\title{
Generalized statistical hydrodynamics of fluids: Approach of generalized collective modes
}

\author{
I.M.Mryglod \\ Institute for Condensed Matter Physics of \\ the National Academy of Sciences of Ukraine \\ 1 Svientsitskii St., 290011 Lviv-11, Ukraine
}

Received June 29, 1998

The aim of this review article is to present recent advances in the generalized hydrodynamics of dense gases and liquids. The presentation attempts to integrate the results known previously with the perspective of the nonequilibrium theory of fluids, but special attention is given to the formalism of generalized collective modes (GCM). This approach allows us to describe consistently the dynamics of fluids in a wide range of wavevectors and frequencies, starting from the hydrodynamic values and up to the Gaussian limit, and to perform the calculation of time correlation functions and generalized transport coefficients within the unified scheme. We start with the basic equations of the theory. Applications of the GCM approach to the studies of dynamical properties of various fluid systems (the LennardJones fluid, metallic Cs, binary mixtures and polar liquids) are then discussed. It is shown how the method can be used in the investigations of magnetic fluids and multicomponent mixtures. Illustrating results, obtained for the GCM spectra of various systems, are presented. Experimental evidence is cited in favour of the observable propagating kinetic modes found in the theory. A limited discussion is given to the mode coupling effects. The relationship to other methods used in the theory of fluids is also discussed.

Key words: generalized hydrodynamics, collective modes, fluids, mixtures, time correlation function

PACS: 05.20.-y, 05.60.+w, 05.70.Ln

In memory of Prof. D.N.Zubarev

\section{Introduction}

Starting from the end of the 60ies, several approaches have been proposed (see, e.g., [1-4] and references therein) for describing the dynamic properties of 
liquids and dense fluids in the intermediate range of wavenumbers $k$ and frequencies $\omega$, where Navier-Stokes hydrodynamics as well as simple methods of kinetic theory could not be applied any more. Phenomenological generalizations of hydrodynamic equations were based mainly on the formal introduction of nonlocal thermodynamic quantities and $(k, \omega)$-dependent transport coefficients and have been used in attempts to bridge the gap between the hydrodynamic ( $\operatorname{small} k, \omega)$, molecular (intermediate $k, \omega$ ) and kinetic (large $k, \omega$ ) regimes. In such a way the semi-phenomenological theories of thermal [5], shear [6] and structural [7] relaxations have been developed. What all these theories have in common is that a new relaxation Maxwell-like time (or, in other words, a new relaxing mode) was taken into consideration and that time was a constant in the hydrodynamic limit. Within the memory function formalism such a modification of the hydrodynamic equations can be formalized by the analysis of the equations for an enlarged multicomponent variable consisting of conserved as well as nonconserved microscopic quantities. It is also worth mentioning that the calculation of higher order corrections in powers of $k$ from either the Navier-Stokes equations or higher order hydrodynamic equations, like the Burnett approximation, is of little use [1] in understanding the behaviour of time correlation functions (TCFs) in the molecular regime and cannot be used at all in the kinetic regime.

In a more rigorous way, the equations for an enlarged set of dynamic variables, including in addition to the hydrodynamic variables the components of microscopic stress tensor and thermal energy flux, were studied for a simple fluid by Akcasu and Daniels [8]. Thereafter this idea was applied to the study of nonequilibrium properties in various fluid systems by several authors (see, e.g., [9]), however, it was not considered in literature [1] as a promising source for the improvement of the results known previously. The most important findings in this way were new interpolation formulas for memory functions $[8,10]$.

The concepts of generalized hydrodynamics have also had a substantial impact on the studies dealing with hard-sphere fluids. In particular, it proves possible to establish an approximate kinetic framework in which the evolution of the main collective modes can be followed in a wide range of wavevectors [11-14]. I was shown that at finite wavenumber $k$ the dynamic structure factor $S(k, \omega)$ can be fitted well by three extended (or generalized) hydrodynamic modes: two extended sound modes and one extended heat mode, which behave as the well-known hydrodynamic modes for small $k$ and display a more complicate $k$-dependence beyond the hydrodynamic range. As a consequence, $S(k, \omega)$ was presented even for nonhydrodynamic wavenumbers as a sum of three Lorentzians with the appropriate amplitudes. Probably, it was the first time when the term an extended or generalized collective mode was rigorously introduced in the theory of fluids as an eigenvalue of the evolution operator.

During the last decade an essential progress in understanding the dynamical properties of fluids was achieved in connection with the method of generalized collective modes (GCM). The concept of generalized collective modes for the study of time correlation functions of a dense monoatomic fluid was initially proposed in 
[14-16]. It is important to note that this approach is based mainly on two physical ideas which were already formulated in the literature. The first idea is to use for the study of generalized hydrodynamics an extended set of dynamic variables containing, in addition to the conserved variables, their higher-order time derivatives. In such a way the kinetic processes which are important for smaller time scales become incorporated into the consideration. And the second one is, in fact, a physical assumption that the Markovian approximation for higher order memory functions can be applied even for nonhydrodynamic values of $k$. Nevertheless, one has to keep in mind that, as it will be seen later, the Markovian approximation for higher order memory functions enables us to study the processes which are essentially non-Markovian from the viewpoint of hydrodynamics. Under these conditions the problem of generalized hydrodynamic description is reduced to the eigenvalues and eigenvectors problem of the generalized hydrodynamic operator, most of the elements of which can be calculated. In paper [16], where the GCM approach within the five-variable description was applied for the first time to the study of a simple fluid with a continuous potential, some elements of the generalized hydrodynamic matrix were considered as adjustable parameters: namely, three $k$-dependent transport coefficients were determined from the weighted leastsquares-fitting procedure as such that gave the best fit to four MD correlation functions, and, in addition, three static correlation functions were also used as adjustable parameters for increasing the accuracy in the fitting procedure. In this way the generalized collective mode spectrum for the Lennard-Jones fluid was calculated and it was shown that all the time correlation functions, constructed on the extended five-component variable, could be fitted consistently by five exponentials that correspond to the five generalized $k$-dependent modes. Thereafter, several attempts have been made to extend the GCM approach to the investigation of more complicated fluid-like systems such as molecular liquids, mixtures and colloidal suspensions (e.g., see $[17,18]$ ). Very promising results have been obtained for binary mixtures. In particular, the theoretical and experimental investigations showed that the dynamic structure factors $S(k, \omega)$ of liquid $\mathrm{Li}_{0.8}-\mathrm{Pb}_{0.2}$ [19], dense gas mixtures He-Ne [20] and He-Ar [21] display a behaviour which can be explained in terms of more than one pair of propagating generalized collective modes only. However, in all the theoretical works listed above the same procedure based on adjustable parameters was used, so that the question whether the GCM approach can be really considered as a successful attempt to construct the generalized hydrodynamic theory remains open.

In [22], the free-parameter generalized collective modes approach, based on the Markovian approximation for the higher order memory functions, was suggested. It was made possible by the use of the explicit relations derived for memory functions. In particular, it was shown that for an arbitrary set of dynamic variables the corresponding memory functions, considered in the Markovian approximation, can be related to the same set of correlation times defined for the hydrodynamic time correlation functions. In [22], the five- and seven-variable descriptions of longitudinal fluctuations for a simple liquid were developed. The extension of the 
formalism to a nine-variable description was presented in [23,24]. The calculation of time correlation functions for the Lennard-Jones fluid $[25,26]$ showed that in a wide range of $k$ and $\omega$ a reasonable agreement of the theory with MD data can be already observed within lower-order mode approximations and the obtained results have an obvious tendency for the convergence to the MD data when the order of mode approximations increases. The generalized $(k, \omega)$-dependent transport coefficients for the Lennard-Jones fluid were calculated within the same approach in [27]. Moreover, it was shown that the developed method is exact in the hydrodynamic limit, and the Gaussian-like behaviour of time correlation functions for large wavenumbers is reproduced with a high degree of accuracy. We note, for example, that the GCM-expression for a dynamic structure factor within the ninemode approximation is written in the form, for which the sum rules are satisfied up to the eighth frequency moment inclusively.

The principal feature of the generalized mode approach, which was absent in the previous attempts to construct the generalized hydrodynamics, is the following: within the GCM approach (apart from the generalizations of thermodynamic quantities and transport coefficients), an extended set of generalized collective modes is studied. Some of them are the so-called generalized kinetic modes, the damping coefficients of which have nonzero values when $k$ is equal to zero. They describe a short time behaviour of the system considered. The other ones can be treated as extended or generalized hydrodynamic collective modes and behave as the well-known hydrodynamic collective modes when wavenumbers are small. Note also that the term a generalized collective mode is used herein in a more general sense, much like in the quantum mechanics, and is not directly associated with the observed peak in a dynamic structure factor, for instance. We treat a generalized collective mode as an eigenvalue of the generalized hydrodynamic operator, so that some of the generalized collective modes cannot be observed experimentally (e.g., because of an overdamped behaviour). On the other hand, it can happen that the generalized kinetic modes, which are not usually observed in the hydrodynamic limit, may become experimentally visible for intermediate wavenumbers $k$ and play even a more important role in the understanding of dynamics than the hydrodynamic ones. To illustrate the last statement, such phenomena as the propagating shear waves $[1,2,25]$, the fast and slow sound in binary mixtures [19-21,30], and spin propagating waves in magnets $[28,29]$ might be recalled, the nature of which can be explained within the GCM treatment. Hence, the generalized kinetic modes, contrary to widely held opinions, have a definite physical significance.

Nowadays, the generalized mode approach represents a modern and powerful method which provides a way for a self-consistent description of dynamical properties practically within the whole range of wavenumbers and frequencies starting from the hydrodynamic values and up to the Gaussian-like range. Within this framework, a time correlation function can be written as a weighted sum of partial terms, each of them, being associated with the relevant generalized collective mode, is expressed via the corresponding eigenvectors and the eigenvalues of the generalized hydrodynamic operator. Using the same approach the generalized 
transport coefficients (including cross correlation ones) can be consistently calculated. Thus, the GCM approach, combined with molecular dynamics simulations, is a very promising tool for the interpretation of experimental data.

In this paper we shall review the progress for the past decade ${ }^{1}$ in a fairly narrow sector prescribed by the limitation of the author's competence and the length of the paper: namely, the GCM approach in the theory of fluids and the comparison of its results with molecular dynamics data and scattering experiments. We do not discuss the advances in the kinetic theory. Only some references to papers in this field will be given in section 5 . Hence, the goal of this paper is: to present in a closed form the basic equations of the generalized collective mode approach derived within a rigorous statistical treatment; to discuss the results obtained by this method for simple fluids (the Lennard-Jones fluid, metallic Cs) and for binary mixtures (HeNe, HeAr, MgZn); and to consider the possibility of subsequent applications of the theory to the study of the dynamic behaviour in magnetic (polar) fluids and in multicomponent mixtures.

The structure of the paper is the following: section 2 outlines the main initial relations and the GCM-expressions which are used in the paper; in section 3 we present the basic equations of the generalized hydrodynamics for a simple fluid; the results and some applications in the theory of binary fluids, magnetic (polar) liquids and multicomponent mixtures are presented in section 4; discussion and concluding remarks are given in section 5 .

\section{Fundamentals}

In order to study the generalized hydrodynamic behaviour of the system considered we have to solve the following problems:

(a) To choose the appropriate set of dynamic variables $\hat{\mathbf{A}}_{0}$ containing conserved variables as well as the relevant nonconserved variables ${ }^{2}$. The latter may be related to the most slow processes in the system which are important for the goal of our study. This set can be extended afterwards by considering the higher order time derivatives of $\hat{\mathbf{A}}_{0}$, so that one obtains a multicomponent dynamic variable $\hat{\mathbf{A}}^{(s)}=\left\{\hat{\mathbf{A}}_{0}, \hat{\mathbf{A}}_{1}, \ldots \hat{\mathbf{A}}_{\mathrm{s}}\right\}$, where $\hat{\mathbf{A}}_{l}=\mathrm{i} \hat{L} \hat{\mathbf{A}}_{l-1}$ and $\mathrm{i} \hat{L}$ is a Liouville operator. The set $\hat{\mathbf{A}}_{0}$ should be specified for each fluid system specifically ${ }^{3}$.

(b) To orthogonalize the dynamic variables $\hat{\mathbf{A}}_{0}$ by introducing new variables $\hat{\mathbf{Y}}_{0}$ which are constructed on the linear combinations of components $\hat{\mathbf{A}}_{0}$ and are orthogonal in a sense that all the static cross correlations for the components of $\hat{\mathbf{Y}}_{0}$ will vanish. This problem has a reason to be considered because of its convenience, and what is more important, the choice of orthogonal variables is directly related

\footnotetext{
${ }^{1}$ We have tried to review the works significant for the topic of this paper. It is certain that we have not found every reference. And of course, our reviews are slightly personal; we took the liberty of expressing some opinions and some ideas of our own.

${ }^{2}$ The explicit dependence of all the quantities on $k$ is omitted for the sake of simplicity.

${ }^{3}$ Note that in some cases it is convenient to define the components of $\hat{\mathbf{A}}_{l}$ for $0<l \leqslant s$ on a subset of $\hat{\mathbf{A}}_{0}$ in order to avoid the appearance of linear dependent components in $\hat{\mathbf{A}}^{(s)}[27,31]$.
} 
to the choice of an appropriate thermodynamic ensemble in which we study our system [32], so that in such a way the generalized thermodynamic quantities can be defined correctly. All the other components of $\hat{\mathbf{A}}^{(s)}$ can be orthogonalized by applying a standard Gram-Schmidt procedure. Hence, one defines a new multicomponent dynamic variable $\hat{\mathbf{Y}}^{(s)}=\left\{\hat{\mathbf{Y}}_{0}, \hat{\mathbf{Y}}_{1}, \ldots, \hat{\mathbf{Y}}_{\mathrm{s}}\right\}$, all the static cross-correlations for which are zero. Note that $\hat{\mathbf{Y}}^{(s)}$ is related to $\hat{\mathbf{A}}^{(s)}$ by a simple equation:

$$
\hat{\mathbf{Y}}^{(s)}=\mathrm{L}^{(s)} \hat{\mathbf{A}}^{(s)},
$$

where $\mathrm{L}^{(s)}$ is a matrix of a linear transformation.

(c) To use one of the methods of the nonequilibrium theory developed for a multicomponent dynamic variable in order to derive transport equations for the averaged components of a dynamic variable; equations for equilibrium time correlation functions (TCFs) constructed on the component of this variable; and an equation for collective excitations. For instance, one may apply the well-known memory function formalism or the framework of the generalized Langevin equation, pioneered by Zwanzig [33] and Mori [34,35].

(d) To find a connection with the Navier-Stokes hydrodynamics in the limit of small $k, \omega$ in order to justify the definitions for the generalized transport coefficients. By solving the transport equations for the averaged components of $\hat{\mathbf{Y}}^{(s)}$ with respect to nonconserved variables, one finds the relations between the hydrodynamic memory functions and the higher order memory functions which can be used either for practical calculations or for deriving extrapolation formulas for the hydrodynamic memory functions.

(e) To calculate consistently the generalized collective spectrum, time correlation functions and the generalized transport coefficients with the help of some approximations for the higher order memory functions, as well as for other needed quantities and to compare the obtained results with the available experimental and/or molecular dynamics data.

Let us start our consideration from the basic equations noted in item (c). The discussion of items (a) and (b) will be reserved for the later section where fluid systems are specified. In our study we applied the method of a nonequilibrium statistical operator $[36,37]$ developed by Zubarev, which has some advantages [38, 39] comparing with other methods known in literature. Within this method the generalized transport equations for a fluid system, which can be used for the study of both strong and weak nonequilibrium cases, were analyzed in [40-42]. In the weak nonequilibrium case for a vector-column of dynamic variables $\hat{\mathbf{P}}$ to be specified later, we found that the transport equations for macroscopic quantities $\langle\Delta \hat{\mathbf{P}}\rangle^{t}$ could be written ${ }^{4}$ in the matrix form known well from the memory function formalism, namely,

$$
\{\mathrm{i} \omega \mathbf{I}-\mathrm{i} \Omega+\tilde{\varphi}(\mathrm{i} \omega+\epsilon)\}\langle\Delta \hat{\mathbf{P}}\rangle^{\omega}=0,
$$

where $\mathbf{I}$ is the unit matrix, $\Delta \hat{\mathbf{P}}=\hat{\mathbf{P}}-\langle\hat{\mathbf{P}}\rangle$, and $\langle\ldots\rangle$ means the equilibrium average. The frequency matrix $\mathrm{i} \Omega$ and the matrix of memory functions $\tilde{\varphi}(z)$ are defined as

\footnotetext{
${ }^{4}$ The angle brackets $\langle\ldots\rangle^{t}$ denote a nonequilibrium averaging, and $\langle\Delta \hat{\mathbf{P}}\rangle^{\omega}$ is the Fourier transform of $\langle\Delta \hat{\mathbf{P}}\rangle^{t}$.
} 
follows:

$$
\begin{gathered}
\mathrm{i} \Omega=\left(\dot{\mathbf{P}}, \Delta \hat{\mathbf{P}}^{+}\right)\left(\Delta \hat{\mathbf{P}}, \Delta \hat{\mathbf{P}}^{+}\right)^{-1} \\
\tilde{\varphi}(z)=\left((1-\mathcal{P}) \dot{\mathbf{P}}, \frac{1}{z+(1-\mathcal{P}) \mathrm{i} \hat{L}}(1-\mathcal{P}) \dot{\mathbf{P}}^{+}\right)\left(\Delta \hat{\mathbf{P}}, \Delta \hat{\mathbf{P}}^{+}\right)^{-1},
\end{gathered}
$$

where $\Delta \hat{\mathbf{P}}^{+}$is the transposed vector, $\dot{\mathbf{P}}=\mathrm{i} \hat{L} \hat{\mathbf{P}}$, and the Mori-like projection operator $\mathcal{P}$ is given by

$$
\mathcal{P} \ldots=\left(\ldots, \Delta \hat{\mathbf{P}}^{+}\right)\left(\Delta \hat{\mathbf{P}}, \Delta \hat{\mathbf{P}}^{+}\right)^{-1} \Delta \hat{\mathbf{P}} .
$$

The matrix equation for the Laplace transform $\tilde{\mathbf{F}}(z)$,

$$
\tilde{\mathbf{F}}(z)=\left(\Delta \hat{\mathbf{P}}, \Delta \hat{\mathbf{P}}^{+}\right)^{z}=\int_{0}^{\infty} \exp \{-z t\} \mathbf{F}(t) \mathrm{d} t,
$$

of the matrix of TCFs $\mathbf{F}(t)$, defined by

$$
\mathbf{F}(t)=\left(\Delta \hat{\mathbf{P}}, \mathrm{e}^{-\mathrm{i} \hat{L} t} \Delta \hat{\mathbf{P}}^{+}\right),
$$

has a structure similar to $(2.2)$, being

$$
\{z \mathbf{I}-\mathrm{i} \Omega+\tilde{\varphi}(z)\} \tilde{\mathbf{F}}(z)=\mathbf{F}(0) .
$$

We note that the matrix elements of $\tilde{\mathbf{F}}(z)$ may be simply expressed in terms of the Fourier transforms of the retarded correlation Green functions

$$
G_{A B}^{(r)}\left(t-t^{\prime}\right)=-\mathrm{i} \theta\left(t-t^{\prime}\right)\left(A(t), B\left(t^{\prime}\right)\right),
$$

where $\theta(t)=1$ or 0 according to either $t>0$ or $t<0$. Hence, the spectrum of the collective modes is determined by the equation

$$
\operatorname{Det}|z I-\mathrm{i} \Omega+\tilde{\varphi}(z)|=0
$$

which gives, in fact, the poles of the regarded correlation Green functions constructed on the set of dynamic variables $\Delta \hat{\mathbf{P}}$.

It should be stressed that the matrix equation for the equilibrium time correlation functions (2.8) is, in fact, an exact equation. This statement can be easily proved using an expression for frequency matrix (2.3) and the alternative expression for the matrix of memory functions in the form:

$$
\begin{array}{r}
\tilde{\varphi}(z)=\left[\left(\dot{\mathbf{P}}, \dot{\mathbf{P}}^{+}\right)^{z}-\left(\dot{\mathbf{P}}, \Delta \hat{\mathbf{P}}^{+}\right)^{z}\left(\left(\Delta \hat{\mathbf{P}}, \Delta \hat{\mathbf{P}}^{+}\right)^{z}\right)^{-1}\left(\Delta \hat{\mathbf{P}}, \dot{\mathbf{P}}^{+}\right)^{z}\right] \\
\times\left(\Delta \hat{\mathbf{P}}, \Delta \hat{\mathbf{P}}^{+}\right)^{-1} .
\end{array}
$$

The transport equations (2.2), the equations for equilibrium time correlation functions (2.8) and the equation for the collective mode spectrum (2.10) form a 
general framework for the study of the dynamical behaviour of fluids in the memory function formalism. In all the expressions given above we used the following definition for a correlation function:

$$
(A, B)=\int_{0}^{1} \mathrm{~d} \tau\left\langle A, \rho_{0}^{\tau} B \rho_{0}^{-\tau}\right\rangle,
$$

where $\rho_{0}$ is an equilibrium statistical operator, so that all the obtained equations can be applied to the study of both quantum and classical systems. It is obvious that $(A, B) \equiv\langle A B\rangle$ in the case of the classical treatment. Note also that the symmetrized expression for the dynamic structure factor of a quantum fluid (see, e.g., $[1-3])$ follows directly from the definition (2.12). Moreover, all the time correlation functions in the expressions written above are presented in a symmetrized form.

Let us consider now the case when $\hat{\mathbf{P}}=\hat{\mathbf{Y}}^{(s)}$ which is, in fact, the first idea of the GCM approach. With such a choice the frequency matrix $i \Omega=i \Omega^{(s)}$ has the following structure [27]:

$$
\mathrm{i} \Omega^{(s)}=\left(\begin{array}{cccccc}
\mathrm{i} \Omega_{0} & \mathcal{I} & & & & \\
-\Gamma_{0} & \mathrm{i} \Omega_{1} & \mathcal{I} & & 0 & \\
& -\Gamma_{1} & \mathrm{i} \Omega_{2} & \mathcal{I} & & \\
& & \ldots & \ldots & \ldots & \\
& 0 & & -\Gamma_{s-2} & \mathrm{i} \Omega_{s-1} & \mathcal{I} \\
& & & & -\Gamma_{s-1} & \mathrm{i} \Omega_{s}
\end{array}\right)
$$

where

$$
\begin{gathered}
\mathrm{i} \Omega_{l}=\mathrm{i} \Omega_{l l}=\left(\mathrm{i} \hat{L} \hat{\mathbf{Y}}_{l}, \hat{\mathbf{Y}}_{l}^{+}\right)\left(\hat{\mathbf{Y}}_{l}, \hat{\mathbf{Y}}_{l}^{+}\right)^{-1} \\
\Gamma_{l}=-\mathrm{i} \Omega_{l+1, l}=-\left(\mathrm{i} \hat{L} \hat{\mathbf{Y}}_{l+1}, \hat{\mathbf{Y}}_{l}^{+}\right)\left(\hat{\mathbf{Y}}_{l}, \hat{\mathbf{Y}}_{l}^{+}\right)^{-1}=\left(\hat{\mathbf{Y}}_{l+1}, \hat{\mathbf{Y}}_{l+1}^{+}\right)\left(\hat{\mathbf{Y}}_{l}, \hat{\mathbf{Y}}_{l}^{+}\right)^{-1}, \\
\mathrm{i} \Omega_{l-1, l}=\left(\mathrm{i} \hat{L} \hat{\mathbf{Y}}_{l-1}, \hat{\mathbf{Y}}_{l}^{+}\right)\left(\hat{\mathbf{Y}}_{l}, \hat{\mathbf{Y}}_{l}^{+}\right)^{-1}=\left(\hat{\mathbf{Y}}_{l}, \hat{\mathbf{Y}}_{l}^{+}\right)\left(\hat{\mathbf{Y}}_{l}, \hat{\mathbf{Y}}_{l}^{+}\right)^{-1}=\mathcal{I}
\end{gathered}
$$

All other block elements of the frequency matrix are equal to zero ${ }^{5}$. From the definitions of memory functions (2.4) and variables $\hat{\mathbf{Y}}_{l}$,

$$
\hat{\mathbf{Y}}_{l}=\left(1-\mathcal{P}_{l-1}\right) \mathrm{i} \hat{L} \hat{\mathbf{Y}}_{l-1}
$$

where

$$
\begin{aligned}
& \mathcal{P}_{l}=\sum_{m=0}^{l} \Delta \mathcal{P}_{m}, \quad \Delta \mathcal{P}_{m}=\left(\ldots, \hat{\mathbf{Y}}_{m}^{+}\right)\left(\hat{\mathbf{Y}}_{m}, \hat{\mathbf{Y}}_{m}^{+}\right)^{-1} \hat{\mathbf{Y}}_{m}, \\
& \mathcal{P}_{0}=\Delta \mathcal{P}_{0}=\left(\ldots, \hat{\mathbf{Y}}_{0}^{+}\right)\left(\hat{\mathbf{Y}}_{0}, \hat{\mathbf{Y}}_{0}^{+}\right)^{-1} \hat{\mathbf{Y}}_{0}
\end{aligned}
$$

\footnotetext{
${ }^{5}$ Of course, one has $i \Omega_{l l}=0$ for the one-component variable $\hat{\mathbf{Y}}_{0}$ because of the symmetry properties.
} 
are Mori-like projection operators, using the equality

$$
\left(1-\mathcal{P}_{\mathrm{s}}\right) \mathrm{i} \hat{L} \hat{\mathbf{P}}=\left(1-\mathcal{P}_{\mathrm{s}}\right)\left(\begin{array}{c}
\mathrm{i} \hat{L} \hat{\mathbf{Y}}_{0} \\
\mathrm{i} \hat{L} \hat{\mathbf{Y}}_{1} \\
\ldots \\
\mathrm{i} \hat{L} \hat{\mathbf{Y}}_{s-1} \\
\mathrm{i} \hat{L} \hat{\mathbf{Y}}_{s}
\end{array}\right)=\left(\begin{array}{c}
0 \\
0 \\
\ldots \\
0 \\
\hat{\mathbf{Y}}_{s+1}
\end{array}\right)
$$

it is easily shown that the matrix of memory functions has only one non-zero block, namely,

$$
\tilde{\varphi}^{(s)}=\left(\begin{array}{ccccc}
0 & 0 & \ldots & 0 & 0 \\
0 & 0 & \ldots & 0 & 0 \\
& & \ldots & & \\
0 & 0 & \ldots & 0 & 0 \\
0 & 0 & \ldots & 0 & \tilde{\varphi}_{\mathrm{s}}
\end{array}\right)
$$

where

$$
\tilde{\varphi}_{\mathrm{s}}(z)=\left(\hat{\mathbf{Y}}_{s+1}, \frac{1}{z+\left(1-\mathcal{P}_{\mathrm{s}}\right) \mathrm{i} \hat{L}} \hat{\mathbf{Y}}_{s+1}^{+}\right)\left(\hat{\mathbf{Y}}_{\mathrm{s}}, \hat{\mathbf{Y}}_{\mathrm{s}}^{+}\right)^{-1}
$$

A set of equations for $\left\{\left\langle\hat{\mathbf{Y}}_{l}\right\rangle^{\omega}\right\}$ follows immediately from (2.2):

$$
\begin{array}{r}
\left(\mathrm{i} \omega \mathcal{I}-\mathrm{i} \Omega_{0}\right)\left\langle\Delta \hat{\mathbf{Y}}_{0}\right\rangle^{\omega}-\left\langle\Delta \hat{\mathbf{Y}}_{1}\right\rangle^{\omega}=0, \\
\Gamma_{0}\left\langle\Delta \hat{\mathbf{Y}}_{0}\right\rangle^{\omega}+\left(\mathrm{i} \omega \mathcal{I}-\mathrm{i} \Omega_{1}\right)\left\langle\Delta \hat{\mathbf{Y}}_{1}\right\rangle^{\omega}-\left\langle\Delta \hat{\mathbf{Y}}_{2}\right\rangle^{\omega}=0, \\
\ldots \ldots \ldots \ldots \ldots \ldots . \ldots \ldots \ldots \\
\Gamma_{s-2}\left\langle\Delta \hat{\mathbf{Y}}_{s-2}\right\rangle^{\omega}+\left(\mathrm{i} \omega \mathcal{I}-\mathrm{i} \Omega_{s-1}\right)\left\langle\Delta \hat{\mathbf{Y}}_{s-1}\right\rangle^{\omega}-\left\langle\Delta \hat{\mathbf{Y}}_{\mathrm{s}}\right\rangle^{\omega}=0, \\
\Gamma_{s-1}\left\langle\Delta \hat{\mathbf{Y}}_{s-1}\right\rangle^{\omega}+\left(\mathrm{i} \omega \mathcal{I}-\mathrm{i} \Omega_{s}+\tilde{\varphi}_{\mathrm{s}}(z)\right)\left\langle\Delta \hat{\mathbf{Y}}_{s}\right\rangle^{\omega}=0 .
\end{array}
$$

Solving the last equation of the set $(2.20)$ with respect to $\left\langle\Delta \hat{\mathbf{Y}}_{s}\right\rangle^{\omega}$ and excluding this variable we obtain the transport equations for the case

$$
\hat{\mathbf{P}}=\hat{\mathbf{Y}}^{(s-1)}=\left\{\hat{\mathbf{Y}}_{0}, \hat{\mathbf{Y}}_{1}, \ldots, \hat{\mathbf{Y}}_{s-1}\right\}
$$

with the memory function $\tilde{\varphi}_{s-1}(z)$ defined by

$$
\tilde{\varphi}_{s-1}(z)=\left(z \mathcal{I}-\mathrm{i} \Omega_{s}+\tilde{\varphi}_{\mathrm{s}}(z)\right)^{-1} \Gamma_{s-1} .
$$

The matrix recurrent relation (2.21) gives a connection between lower- and higherorder memory functions and can be used for an arbitrary value of $s \geqslant 1$. It should be stressed that relation (2.21) is also exact. Repeating the same procedure one can find the transport equation for the set $\left\langle\hat{\mathbf{Y}}_{0}\right\rangle$ with the same structure as equation $(2.2)$ and the memory function $\tilde{\varphi}_{0}(z)$. The explicit form for $\tilde{\varphi}_{0}(z)$ via $\tilde{\varphi}_{l}(z)$ with $0<l \leqslant s$ and the higher order elements of frequency matrix (2.13) follow from the recurrent relation (2.21). Thus, up to this place our consideration of 
an extended set of dynamic variable may be treated as some mathematical trick which is of little use. However, one should point out that an explicit representation for $\tilde{\varphi}_{0}(z)$ is obtained, which reflects the analytic structure of the memory function. Note also that for $s \geqslant 1$ function $\tilde{\varphi}_{0}(z)$ depends on $z$ even if the matrix of higher order memory functions $\tilde{\varphi}_{s}(z)$ is taken in the Markovian approximation $\tilde{\varphi}_{s}(z) \simeq \tilde{\varphi}_{s}(0)$.

Starting from this point there exist two main ways for developing the theory. The first one, widely used in the common practice of the memory function formalism [1-4], consists in choosing some extrapolation formula for $\tilde{\varphi}_{0}(z)$ (Markovian-, Lorentzian-, Gaussian-, two-exponential-like approximations, etc.) depending on the physics to be studied, so that our consideration is restricted by the equations for the set $\hat{\mathbf{Y}}_{0}{ }^{6}$. The second possibility is the following: we use the most simple Markovian approximation for the matrix $\tilde{\varphi}_{s}(z)$ and consider equations for the extended set $\hat{\mathbf{Y}}^{(s)}$. It is obvious that in such a case the results for TCFs, constructed on $\hat{\mathbf{Y}}_{0}$, are to be the same as the results obtained by the first method when the extrapolation formula is constructed on the basis of relation (2.21). In this sense both methods are quite equivalent. However, the second way has some additional advantages in comparison with the first one. In order to clarify this statement let us consider the next steps in the GCM approach and use the Markovian approximation for the highest order memory function $\tilde{\varphi}_{\mathrm{s}}(z)$, assuming that $\tilde{\varphi}_{\mathrm{s}}(z) \simeq \tilde{\varphi}_{\mathrm{s}}(0)$. In this case we find that the equation for $\tilde{\mathbf{F}}(z) \equiv\left(\Delta \hat{\mathbf{P}}, \Delta \hat{\mathbf{P}}^{+}\right)^{z}$ with $\hat{\mathbf{P}}=\hat{\mathbf{Y}}^{(s)}$ has the most simple form, namely,

$$
\left\{z \mathbf{I}+\mathbf{T}^{(s)}\right\} \tilde{\mathbf{F}}^{(m, s)}(z)=\mathbf{F}^{(s)}(0),
$$

where

$$
\mathbf{T}^{(s)}=-\mathrm{i} \Omega^{(s)}+\tilde{\varphi}^{(s)}(0)
$$

is a generalized hydrodynamic matrix within the $s$ th order approximation. Using definition (2.11) it can be shown [22] that an alternative expression for matrix $\mathbf{T}^{(s)}$ is also valid:

$$
\mathbf{T}^{(s)}=\mathbf{F}^{(s)}(0)\left[\tilde{\mathbf{F}}^{(s)}(0)\right]^{-1} .
$$

It will be seen later that this representation for $\mathbf{T}^{(s)}$ is very useful for concrete applications.

The solution of equation (2.22) can be written in an analytical form via the eigenvalues $z_{\alpha}$ and eigenvectors $\hat{\mathbf{X}}_{\alpha}=\left\|\hat{\mathrm{X}}_{i, \alpha}\right\|$ of the matrix $\mathbf{T}^{(s)}=\left\|\mathrm{T}_{i j}\right\|$

$$
\sum_{j=1}^{M} \mathrm{~T}_{i j} \hat{\mathrm{X}}_{j, \alpha}=z_{\alpha} \hat{\mathrm{X}}_{i, \alpha}
$$

where $i, j=1, \ldots, M$ and $M$ is a total number of components taken into account in the multicomponent vector $\hat{\mathbf{Y}}^{(s)}$. For the elements $\tilde{\mathrm{F}}_{i j}^{(m, s)}(z)$ of the matrix $\tilde{\mathrm{F}}^{(m, s)}(z)$

\footnotetext{
${ }^{6}$ It should be stressed that the recurrent relations like equation (2.21) can be very useful even in this case, because they provide a correct way for constructing extrapolation formulas.
} 
one gets

$$
\tilde{\mathrm{F}}_{i j}^{(m, s)}(z)=\sum_{\alpha=1}^{M} \frac{G_{\alpha}^{i j}}{z+z_{\alpha}},
$$

so that

$$
\mathrm{F}_{i j}^{(m, s)}(t)=\sum_{\alpha=1}^{M} G_{\alpha}^{i j} \exp \left\{-z_{\alpha} t\right\},
$$

where the weight coefficients $G_{\alpha}^{i j}$ are given by the expression

$$
G_{\alpha}^{i j}=\sum_{l=1}^{M} \hat{\mathrm{X}}_{i, \alpha} \hat{\mathrm{X}}_{\alpha, l}^{-1} \mathrm{~F}_{l j}(0) .
$$

Matrix $\hat{\mathbf{X}}^{-1}$ is the inverse of $\hat{\mathbf{X}}=\left\|\hat{\mathbf{X}}_{\alpha}\right\|$.

Hence, functions $\mathrm{F}_{i j}^{(m, s)}(t)$ are presented as a sum of $M$ exponential terms, and each term is related to the corresponding effective collective mode $z_{\alpha}$. The amplitude $G_{\alpha}^{i j}$ describes a partial contribution associated with eigenvalue $z_{\alpha}$ in the $\mathrm{TCF} \mathrm{F}_{i j}^{(m, s)}(t)$. It is important to note that all unknown quantities in (2.26) depend only on $k$. The expression (2.26) is valid for any $s$ and can be considered as quite a general formulation of the results known previously [16] (see also [1,2]).

One of the most significant advantages of the GCM approach is that the problem of fluid dynamics is reduced to the eigenvectors and eigenvalues problem well studied in mathematics. This makes it possible to use a lot of methods developed in this field. Another important finding is that the higher order sum rules for the time correlation functions constructed on the set $\hat{\mathbf{Y}}_{0}$ are performed up to $(2 s+2)$ th order inclusively ${ }^{7}$. Moreover, the zero order time moments of these functions are reproduced explicitly as well. More generally one can say that within the sth order approximation of the GCM approach, at least the first $(2 s+2)$ moments (either in time- or frequency-spaces) for each of the $M \times M$ time correlation functions $\tilde{\mathbf{F}}^{(m, s)}(z)$ coincide with the explicit values of the moments associated with the corresponding genuine function from $\tilde{\mathbf{F}}^{(s)}(z)[27,43]$. That is why the Gaussian-like behaviour of TCFs in the limit of large wavenumbers must be described correctly within this approach. In practice, this means that the Gaussian line shape can be mimiced very well by $M$ exponentials when $M$ is large enough (e.g., $M$ larger than three $[16,25-27])$.

To conclude this section we will discuss briefly what makes the generalized mode approach especially attractive for using it together with computer simulations. First, taking into account the relation between the sets of variables $\hat{\mathbf{A}}^{(s)}$ and $\hat{\mathbf{Y}}^{(s)}$ (see equation $(2.1)$ ), it is easy to show that all the results given above by (2.22)-(2.26) can be rewritten for the matrix of time correlation functions $\mathcal{F}^{(s)}(t)$,

$$
\mathcal{F}^{(s)}(t)=\left(\hat{\mathbf{A}}^{(s)}, \mathrm{e}^{-\mathrm{i} \hat{L} t} \hat{\mathbf{A}}^{(s)}\right),
$$

\footnotetext{
${ }^{7}$ As it will be seen later within the $s$ th order approximation the sum rules for the dynamic structure factor are reproduced up to the $(2 s+4)$ th order inclusively $[27,43]$.
} 
defined for the set $\hat{\mathbf{A}}^{(s)}$. One finds the relations

$$
\begin{aligned}
& \mathbf{F}^{(s)}(t)=\mathrm{L}^{(s)} \mathcal{F}^{(s)}(t)\left[\mathrm{L}^{(s)}\right]^{+}, \quad \tilde{\mathbf{F}}^{(s)}(z)=\mathrm{L}^{(s)} \tilde{\mathcal{F}}^{(s)}(z)\left[\mathrm{L}^{(s)}\right]^{+}, \\
& \mathbf{T}^{(s)}=\mathrm{L}^{(s)} \mathcal{T}^{(s)}\left[\mathrm{L}^{(s)}\right]^{-1}, \quad \hat{\mathbf{X}}=\mathrm{L}^{(s)} \hat{\mathcal{X}}, \quad \mathbf{G}_{\alpha}=\mathrm{L}^{(s)} \mathcal{G}_{\alpha}\left[\mathrm{L}^{(s)}\right]^{+},
\end{aligned}
$$

where $\mathcal{T}^{(s)}, \hat{\mathcal{X}}$, and $\mathcal{G}_{\alpha}$ are the generalized hydrodynamic matrix, the matrix of eigenvectors, and the matrices of weight coefficients defined on the set $\hat{\mathbf{A}}^{(s)}$, respectively. Note that $\mathcal{T}^{(s)}=\mathcal{F}^{(s)}(0)\left[\tilde{\mathcal{F}}^{(s)}(0)\right]^{-1}$ and all the quantities in this expression can be calculated by molecular dynamics either by taking the time derivatives from the hydrodynamic time correlation functions [16] or for classical fluids directly in computer simulations $[22,30]$. They are only static correlation functions and the correlation times for hydrodynamic TCFs constructed on the subset $\hat{\mathbf{A}}_{0}$. Second, it will be seen later that the elements of matrix $\mathrm{L}^{(s)}$ can be also expressed via the elements of matrix $\mathcal{F}^{(s)}(0)$, so that one has a way for self-consistent calculations of matrix $\mathbf{T}^{(s)}$ or, in other words, matrices $\mathrm{i} \Omega^{(s)}$ and $\tilde{\varphi}^{(s)}(0)$ applying equations (2.29). Therefore, the generalized transport coefficients, related to the higher order memory functions by equation (2.21), are determined within the same scheme.

Another point which is worth mentioning is the following: as it follows from (2.29), the generalized hydrodynamic matrix $\mathbf{T}^{(s)}$ is related to $\mathcal{T}^{(s)}$ by a unitary transformation. This means that the sets of eigenvalues for both matrices $\mathbf{T}^{(s)}$ and $\mathcal{T}^{(s)}$ are the same and this result is independent of the statistical ensemble used for the description. Hence, the spectrum of generalized collective modes has a significant physical meaning (in some sense even more important than, for instance, the weight coefficients or time correlation functions themselves). The same collective excitations will contribute, in principle, to each of the time correlation functions, and an amount of this contribution is to be determined by the corresponding weight coefficient ${ }^{8}$.

Therefore, an algorithm of our study within the GCM approach is the following:

(1) to calculate the static correlation functions and the correlation times needed and to find the generalized hydrodynamic operator $\mathcal{T}^{(s)}$;

(2) to solve the eigenvalue problem for $\mathcal{T}^{(s)}$ and to determine the eigenvalues $\left\{z_{\alpha}\right\}$ and the eigenvectors $\left\{\hat{\mathcal{X}}_{\alpha}\right\}$

(3) to calculate the time correlation functions using the analytic solutions for them (see (2.26) and (2.27)). Note that separated contributions of each of the collective modes can be obtained;

(4) to find matrix $\mathrm{L}^{(s)}$ and, using then equations (2.29), (2.23), (2.21) and (2.13), to calculate the generalized transport coefficients.

Hence, the needed input information includes $k$-dependent static correlation functions as well as the correlation times, and the output of the theory is: the generalized collective mode spectrum; $M \times M$ time correlation functions in which partial contributions from collective excitations can be separated; and the generalized

\footnotetext{
${ }^{8}$ Of course, some of the weight coefficients can be equal to zero because of the symmetry reasons, and in such a case the relative collective mode has no contribution to a time correlation function.
} 
$(k, \omega)$-dependent transport coefficients. Let us consider now some realistic models of fluids and analyze the results obtained for them within the GCM approach.

\section{Generalized hydrodynamics of a simple fluid}

\subsection{Transverse fluctuations}

We consider first a description of transverse fluctuations in simple classical fluids in order to specify the basic ideas of the GCM approach and to compare the obtained results with the ones known previously. Moreover, this example may be considered as traditional in the attempts to apply various theories of generalized hydrodynamics. The main objective herein is to describe the appearance of propagating excitations associated with shear waves in liquids, beyond the hydrodynamic region when $k$ becomes lager than some cutoff wavenumber $k_{\mathrm{H}}[1-4]$. A large amount of MD data obviously showed that there exists a propagating gap with $0<k<k_{\mathrm{H}}$ within which a well-known hydrodynamic behaviour takes place, so that shear waves cannot propagate. Otherwise, for $k>k_{\mathrm{H}}$, a fluid behaves like a viscoelastic medium where the transverse shear waves can travel through the fluid, like in solids. This crossover is a good example of the dynamical properties in which an interplay between long (hydrodynamic) and short time processes is reflected.

The equation of generalized hydrodynamics for transverse fluctuations can be written as follows $[1,3]$ :

$$
\mathrm{i} \omega\left\langle\hat{J}_{k}^{t}\right\rangle^{\omega}+\tilde{\varphi}_{J J}^{(t)}(k, \mathrm{i} \omega+\epsilon)\left\langle\hat{J}_{k}^{t}\right\rangle^{\omega}=0, \quad \epsilon \rightarrow 0,
$$

where

$$
\hat{J}_{k}^{t}=\frac{1}{\sqrt{N}} \sum_{i=1}^{N} p_{i}^{t} \exp \left\{\mathrm{i} k z_{i}\right\}
$$

is a transverse component of the current. In equations (3.1) and (3.2) we suppose that wavevector $\mathbf{k}(k \neq 0)$ is directed along the $0 Z$ axis and $p_{i}^{t}$ is a projection of the $i$-th particle momentum onto a direction perpendicular to this axis. $\tilde{\varphi}_{J J}^{(t)}(k, z)$ is the Laplace transform of the hydrodynamic memory function $\varphi_{J J}^{(t)}(k, t)$,

$$
\varphi_{J J}^{(t)}(k, t)=\left\langle\mathrm{i} L_{N} J_{k}^{t} \exp \left\{-\left(1-\mathcal{P}_{0}\right) \mathrm{i} L_{N}\right\} \mathrm{i} L_{N} J_{-k}^{t}\right\rangle\left\langle J_{k}^{t} J_{-k}^{t}\right\rangle^{-1},
$$

and

$$
\mathcal{P}_{0} \ldots=\left\langle\ldots \hat{J}_{-k}^{t}\right\rangle\left\langle\hat{J}_{k}^{t} \hat{J}_{-k}^{t}\right\rangle^{-1} \hat{J}_{k}^{t}
$$

is the Mori-like projection operator.

The generalized shear viscosity $\tilde{\eta}(k, z)$ is simply connected with the memory function $\tilde{\varphi}_{J J}^{(t)}(k, z)$ by the relation

$$
\tilde{\eta}(k, z)=\frac{\rho}{k^{2}} \tilde{\varphi}_{J J}^{(t)}(k, z)=\frac{\rho}{k^{2}}\left(-z+\frac{f_{J J}^{(t)}(k)}{\tilde{f}_{J J}^{(t)}(k, z)}\right),
$$


and

$$
\lim _{\omega \rightarrow 0} \lim _{k \rightarrow 0} \operatorname{Re} \eta(k, \mathrm{i} \omega+\epsilon)=\eta,
$$

where $\rho=m n=m N / V$ is the mass density, and $\eta$ is the shear viscosity coefficient. As it is well-known, $\eta$ can be written in the form of a Green-Kubo formula [1-4].

An extended set of dynamic variables can be defined as follows:

$$
\hat{\mathbf{A}}^{(s)}=\left\{\hat{A}_{0}, \hat{A}_{1}, \ldots, \hat{A}_{\mathrm{s}}\right\},
$$

where $\hat{A}_{l}(k)=(\mathrm{i} \hat{L})^{l} \hat{J}_{k}^{t}, l=0,1, \ldots, s$, so that all the general expressions obtained above can be directly applied to this case. Matrix $\mathcal{T}^{(s)}(k)$ defined in terms of initial variables $\hat{\mathbf{A}}^{(s)}$ can be written in the form:

$$
\mathcal{T}^{(s)}(k)=\mathcal{F}^{(s)}(k) / \tilde{\mathcal{F}}(k),
$$

where $\mathcal{F}^{(s)}(k)=\left\langle\hat{\mathbf{A}}^{(s)}(k)\left[\hat{\mathbf{A}}^{(s)}(k)\right]^{+}\right\rangle$is a matrix of static correlation functions and $\tilde{\mathcal{F}}^{(s)}(k)=\tilde{\mathcal{F}}^{(s)}(k, z=0)$, being

$$
\tilde{\mathcal{F}}^{(s)}(k, z)=\int_{0}^{\infty} \mathrm{d} t \mathrm{e}^{-z t} \mathcal{F}^{(s)}(k, t) .
$$

It can be shown $[22,25]$ that $\mathcal{F}^{(s)}(k)$ is a real symmetric matrix and has the following structure:

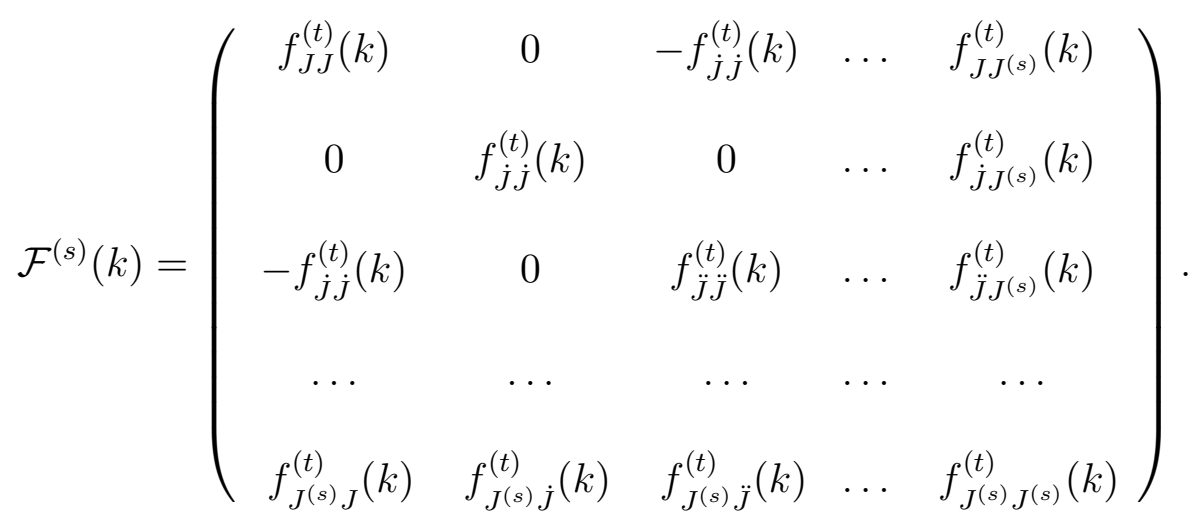

Matrix $\tilde{\mathcal{F}}^{(s)}(k)$ is antisymmetrical and can be written as follows:

$$
\tilde{\mathcal{F}}^{(s)}(k)=\left(\begin{array}{ccccc}
\tau_{J J}^{(t)}(k) f_{J J}^{(t)}(k) & f_{J J}^{(t)}(k) & 0 & \ldots & f_{J J^{(s-1)}}^{(t)}(k) \\
-f_{J J}^{(t)}(k) & 0 & f_{\dot{J} \dot{J}}^{(t)}(k) & \ldots & f_{J^{(s-1)}}^{(t)}(k) \\
0 & -f_{\dot{J} j}^{(t)}(k) & 0 & \ldots & f_{J^{(s-1)}}^{(t)}(k) \\
\ldots & \ldots & \ldots & \ldots & \ldots \\
-f_{J^{(s-1)} J}^{(t)}(k) & -f_{J^{(s-1) j}}^{(t)}(k) & -f_{J^{(s-1)} j}^{(t)}(k) & \ldots & -f_{J^{(s-1)} J^{(s)}}^{(t)}(k)
\end{array}\right) .
$$


We used the notations $f_{i j}(k, t)$ for the TCFs defined on the set of initial variables $\left\{\hat{A}_{l}(k)\right\}$, and $i, j=\left\{J, \dot{J}, \ddot{J}, \ldots, J^{(s)}\right\}$. Note that in the expressions for $\mathcal{F}^{(s)}(k)$ and $\tilde{\mathcal{F}}^{(s)}(k)$ : (i) $s$ takes an arbitrary value $(s \geqslant 0)$; (ii) each second element in the last line, as well as in the last column depending on whether $s$ is even or odd, is equal to zero because of the symmetry properties; and (iii) all the matrix elements are static correlation functions and can be calculated using the analytic expressions for the sum rules of the current-current transverse time correlation function $f_{J J}^{(t)}(k, t)$ (see, e.g., $[1,2,44])$ or directly in computer simulations $[22,25]$. One exception is only the value of $\tau_{J J}^{(t)}(k)$ which is originally a dynamical quantity known as the transverse correlation time

$$
\tau_{J J}^{(t)}(k)=\frac{1}{f_{J J}^{(t)}(k)} \int_{0}^{\infty} \mathrm{d} t f_{J J}^{(t)}(k, t) .
$$

As it is seen from $(3.8), \tau_{J J}^{(t)}(k)$ can be also calculated by molecular dynamics. We note that recently a new iterative procedure for the calculation of this quantity based on the knowledge of static correlation functions was developed [45,50], and it was shown that this procedure is well convergent to MD data.

The representation (3.7) for the matrix $\mathcal{T}^{(s)}(k)$ is very convenient for subsequent calculations. As it is apparent from its structure, in order to improve the results for the approximated TCFs within the GCM approach, it is just necessary to include into consideration the higher-order static correlation functions.

Within the sth order approximation of the GCM approach, the generalized shear viscosity $\tilde{\eta}^{(s)}(k, z)$ can be found with the help of (3.5), taking instead of the genuine function $\tilde{f}_{J J}^{(t)}(k, z)$ a solution for the Laplace transform of the transverse current-current time correlation functions obtained in this approximation.

As an illustration, the spectra of generalized transverse collective modes, calculated $[22,24]$ for a Lennard-Jones fluid in two- $(s=1)$ and four-variables $(s=3)$ approximations $^{9}$ of the GCM approach, are shown in figure 1.

We see in figure 1 that:

(i) There are two types of generalized collective modes. Of all the eigenvalues of $\mathcal{T}^{(s)}(k)$, only one eigenvalue has the property showing that the damping vanishes when $k$ tends to zero. Moreover, in the hydrodynamic limit this eigenvalue is purely diffusive (see figure 1a) and has the same asymptotic behaviour independently of the order of the approximation used. This is a generalized transverse hydrodynamic mode which has the well-known asymptote of $D k^{2}[2,1]$, when $k \rightarrow 0$, and $D=\eta / \rho$ is the kinematic shear viscosity.

(ii) All the other eigenvalues approach finite damping coefficients, when $k$ tends to zero. They are generalized kinetic modes. Note also that for small and intermediate wave-numbers of $k$ a tendency for converging the results can be observed when the order of mode approximations increases.

(iii) The generalized collective modes have the complex-conjugate eigenvalues $z_{t}(k)=\sigma_{t}(k) \pm \mathrm{i} \omega_{t}(k)$ practically for all the wave-numbers $k$ considered. One

\footnotetext{
${ }^{9}$ The results for even $s$ have some specific features due to the time symmetry properties $[22,24]$.
} 

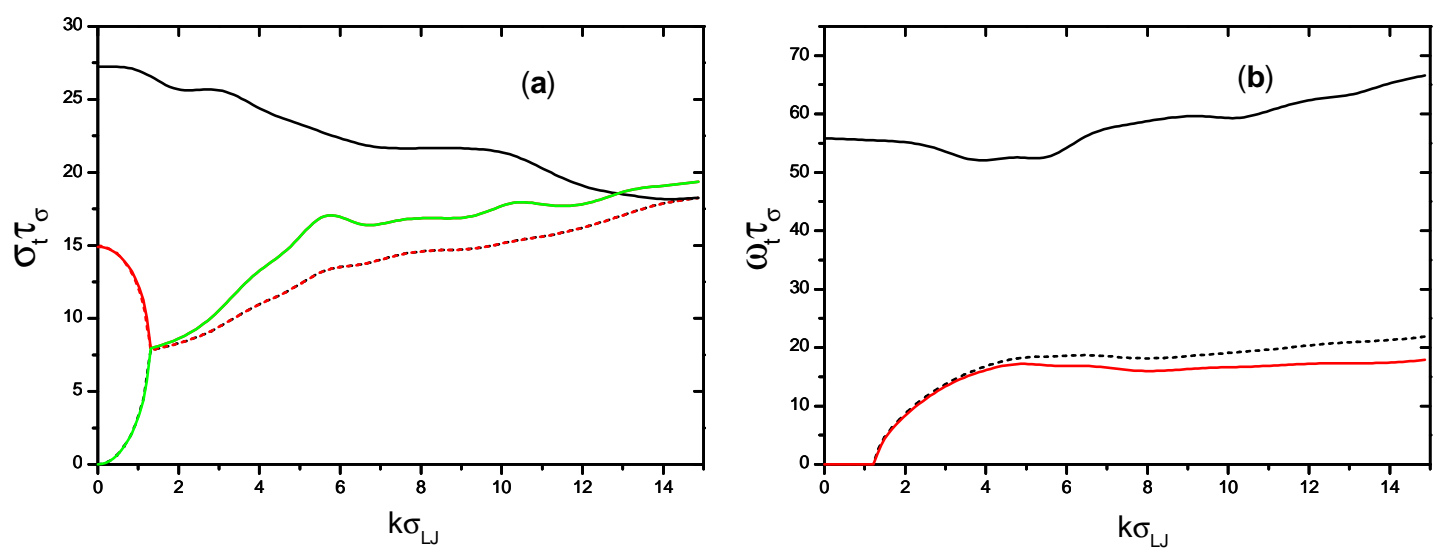

Figure 1. Generalized transverse mode spectrum of the LJ fluid at $n^{*}=0.845$ and $T^{*}=1.705$ : (a) damping coefficients and (b) dispersions. The results of twoand four-mode approximations are plotted by dashed and solid lines, respectively. From [24].

exception is found only for the lowest-lying kinetic mode, when $k \sigma_{\mathrm{LJ}}<1.25$ (dashed and solid curves in figure 1b).

Thus, as it is seen in figure $1 \mathrm{~b}$, when $k$ increases (starting from the hydrodynamic values) the generalized hydrodynamic mode and the lowest-lying relaxing kinetic mode mix and form at $k_{\mathrm{H}} \simeq 1.25 \sigma_{\mathrm{LJ}}^{-1}$ a new pair of propagating complexconjugated modes. These modes are responsible for the propagating of shear waves in a fluid, when $k$ becomes larger than $k_{\mathrm{H}}$, so that the wavenumber $k_{\mathrm{H}}$ characterizes the width of the propagating gap discussed at the beginning of this section. The results obtained [25] for the transverse current-current time correlation and generalized shear viscosity have strengthened our viewpoint. The higher-lying propagating kinetic modes will be discussed in the next section in comparison with other high-frequency modes observed in fluids.

The minimum set of dynamic variables within which the propagating gap could be described is $\hat{\mathbf{A}}^{(1)}=\left\{J_{k}^{t}, \mathrm{i} \hat{L} \hat{J}_{k}^{t}\right\}$, where $\mathrm{i} \hat{L} J_{k}^{t}=-\mathrm{i} k \hat{\sigma}_{k}^{t}$ and $\hat{\sigma}^{t}$ is a transverse component of the microscopic stress tensor. Considering the generalized transport equations (see (2.22)) for this case and assuming that $k$ is small, we get

$$
\begin{array}{r}
\mathrm{i} \omega\left\langle\hat{J}_{k}^{t}\right\rangle^{\omega}+\mathrm{i} k\left\langle\hat{\sigma}_{k}^{t}\right\rangle^{\omega}=0, \\
\mathrm{i} k \gamma_{0}\left\langle\hat{J}_{k}^{t}\right\rangle^{\omega}+\mathrm{i} \omega\left\langle\hat{\sigma}_{k}^{t}\right\rangle^{\omega}+\frac{1}{\tau_{1}}\left\langle\hat{\sigma}_{k}^{t}\right\rangle^{\omega}=0,
\end{array}
$$

where $\gamma_{0}=\Gamma_{0}(k) /\left.k^{2}\right|_{k \rightarrow 0}=G / \rho, \tau_{1}=1 /\left.\tilde{\varphi}_{1}(k, 0)\right|_{k \rightarrow 0}=\eta / G$, and $G$ is the rigidity modulus. Solving the first equation of the set (3.9) with respect to $\left\langle\hat{J}_{k}^{t}\right\rangle^{\omega}$ one obtains

$$
\left(\omega^{2}-\gamma_{0} k^{2}\right)\left\langle\hat{\sigma}_{k}^{t}\right\rangle^{\omega}=\frac{\mathrm{i} \omega}{\tau_{1}}\left\langle\hat{\sigma}_{k}^{t}\right\rangle^{\omega}
$$

Equation (3.10) is, in fact, a telegraph equation describing a transmission of waves with the speed $c=\sqrt{\gamma_{0}}$ and was used in literature for the study of shear waves in a continuous medium (see, e.g., [46] and references therein). Using (3.9) the value 
of $k_{\mathrm{H}}$ can be easily estimated from the condition that at $k=k_{\mathrm{H}}$ the propagating collective excitations appear. One gets for the width of the propagating gap

$$
k_{\mathrm{H}} \simeq \frac{\sqrt{G \rho}}{2 \eta}
$$

in full agreement ${ }^{10}$ with the viscoelastic theory $[1,2]$, and $\tau_{1}$ is the Maxwell relaxation time in the terminology of the viscoelastic theory.

We present here this simple analysis in order to show that the GCM approach correlates well with both phenomenological [46] and memory function $[1,2]$ treatments. Within the next approximation $(s=2)$ the higher order extrapolation formulas (see, e.g., [8]) for memory functions can be reproduced. Moreover, as it will be seen later, such a consideration is very useful for understanding the behaviour of collective modes describing the longitudinal fluctuations as well.

The time correlation functions and the generalized shear viscosity for the Lennard-Jones fluid at $n^{*}=0.845$ and $T^{*}=1.705$ were calculated within the fourvariable approximation $(s=2)$ in [25]. We found that the results of the fourmode description are very close to MD data for wavenumbers varying within wide limits from small (hydrodynamic) values and up to the Gaussian-like range. Similar results have been obtained for metallic Cs in [51], where it was shown that the specific behaviour of transverse excitations in metallic Cs near the melting point is conditioned by an extremely narrow propagating gap.

In [45], we studied the so-called shoulder problem recognized for the $k$-dependent shear viscosity at intermediate wavevectors by Balucani et al. [4,47]. The problem is as follows: some discrepancies persist at intermediate wavenumbers, where a sort of shoulder in the data cannot be reproduced by any of the phenomenological models. Recalling that within the GCM approach the transverse correlation time $\tau_{J J}^{(t)}(k)$ (or, in other words, the $k$-dependent shear viscosity $\eta(k)=$ Re $\left.\tilde{\eta}(k, 0)=\rho / \tau_{J J}^{(t)}(k)\right)$ can be considered as an input parameter of the theory, it was necessary to use some approximated procedure for the calculation of $\tau_{J J}^{(t)}(k)$ and to compare then the results to be obtained with MD data. Such a procedure was developed $[45,50]$ and was based on the assumption that the higher order relaxation times $\tau_{\mathrm{s}}(k)=1 / \tilde{\varphi}_{\mathrm{s}}(k, 0)$ have a tendency for saturation when $s$ increases, so that $\tilde{\varphi}_{s+1}(k, 0) \simeq \tilde{\varphi}_{\mathrm{s}}(k, 0)$ for $s \gg 1$. Under this condition we used the recurrent relations (2.21) for memory functions and calculated the transverse correlation time $\tau_{J J}^{(t)}(k)$. The results obtained for $\eta(k)$ showed good agreement with MD data for all the wavenumbers considered, including the intermediate values.

\subsection{Longitudinal fluctuations}

For longitudinal fluctuations a set of dynamic variables can be chosen in the form $[22,26,27]$ :

$$
\hat{\mathbf{A}}^{(s)}(k)=\left\{\hat{\mathbf{A}}_{0}(k), \hat{\mathbf{A}}_{1}(k), \ldots, \hat{\mathbf{A}}_{\mathbf{s}}(k)\right\},
$$

\footnotetext{
${ }^{10}$ Note that in the viscoelastic theory a critical value of $k_{\mathrm{c}}$ defined from the resonant condition for the Fourier transform of the transverse current TCF function is usually considered, so that $k_{\mathrm{c}}=\sqrt{2} k_{\mathrm{H}}$.
} 
where $\hat{\mathbf{A}}_{l}(k)=\mathrm{i} \hat{L}^{l} \hat{\mathcal{A}}(k), l=1,2, \ldots, s, \hat{\mathbf{A}}_{0}(k)=\left\{\hat{n}_{k}, \hat{\mathcal{A}}(k)\right\}$, and $\hat{\mathcal{A}}(k)=\left\{\hat{J}_{k}, \hat{e}_{k}\right\}$. The variables $\hat{n}_{k}, \hat{J}_{k}$, and $\hat{e}_{k}$ denote the densities of particles' number, longitudinal current and energy, respectively.

All the elements of matrices $\mathcal{F}^{(s)}(k)$ and $\tilde{\mathcal{F}}^{(s)}(k, 0)$ can be expressed [22] via the static correlation functions $f_{i j}(k)$ and the three correlation times $\tau_{\alpha \beta}(k)$ where $f_{i j}(k)=\left\langle A_{i}(k) A_{j}(-k)\right\rangle$ with $i, j=\{1,2, \ldots, M\}, M=2 s+3$,

$$
\hat{\mathbf{A}}^{(s)}(k)=\left\{\hat{A}_{1}(k), \hat{A}_{2}(k), \ldots, \hat{A}_{M}(k)\right\}=\left\{\hat{n}_{k}, \hat{J}_{k}, \hat{e}_{k}, \dot{J}_{k}, \dot{e}_{k}, \ldots, \mathrm{i} L_{N}^{s} \hat{J}_{k}, \mathrm{i} L_{N}^{s} \hat{e}_{k}\right\}
$$

and

$$
\tau_{\alpha \beta}(k)=\frac{1}{f_{\alpha \beta}(k)} \int_{0}^{\infty} \mathrm{d} t f_{\alpha \beta}(k, t)=\frac{\tilde{f}_{\alpha \beta}(k, 0)}{f_{\alpha \beta}(k)},
$$

with $\alpha, \beta=\{n, J, e\}$.

In the nine-mode approximation $(s=3)$ matrix $F^{(s)}(k)$ has the form:

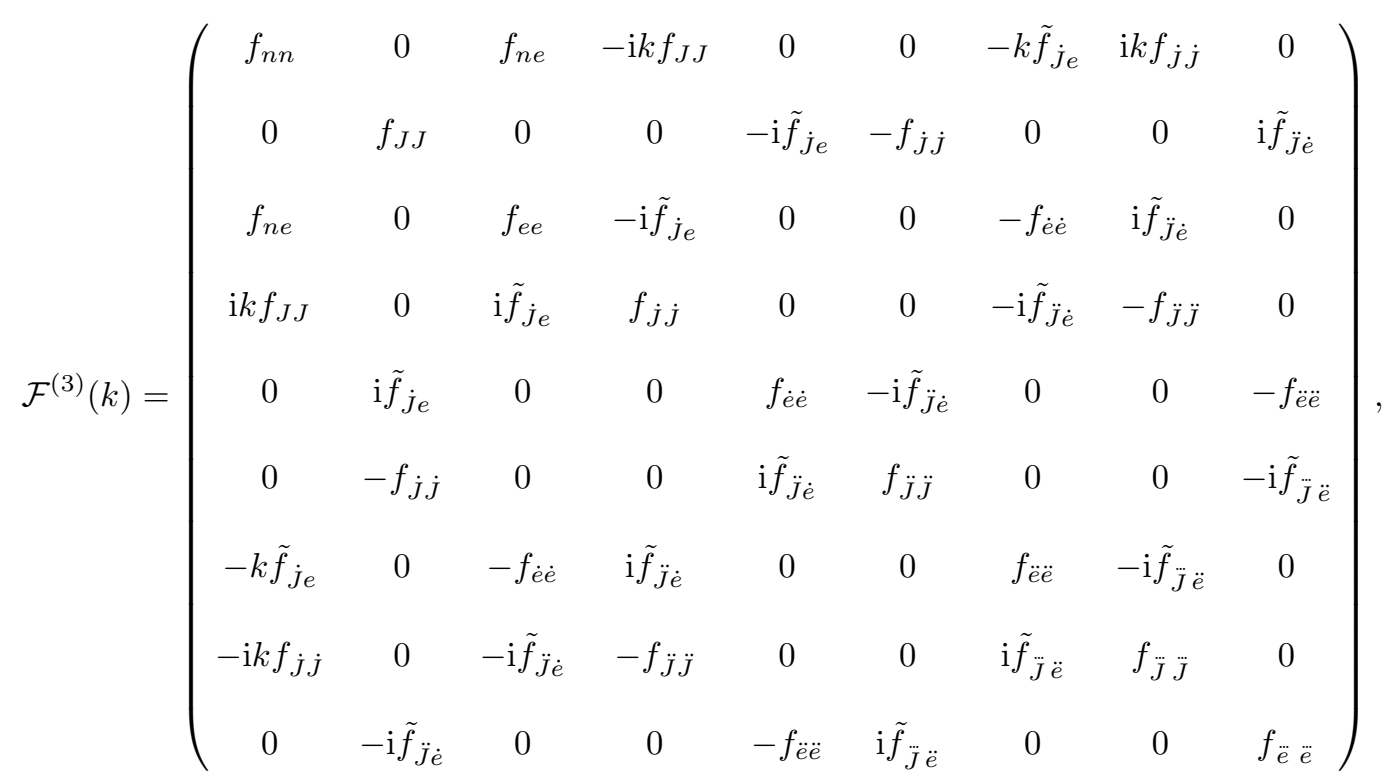

where it was set $m=1$ for the brevity sake. Some of the static correlation functions are purely imaginary, namely, $f_{\dot{J} e}(k)=\mathrm{i} \tilde{f}_{\dot{J} e}(k), f_{\ddot{J} \dot{e}}(k)=\mathrm{i} \tilde{f}_{\ddot{J} \dot{e}}(k)$, and $f_{\ddot{J} \ddot{e}}(k)=$ $\mathrm{i} \tilde{f}_{\bar{J}} \ddot{e}(k)$. Note that $\mathcal{F}^{(s)}(k)$ is a Hermitian matrix. 
In the same approximation matrix $\tilde{\mathcal{F}}^{(s)}(k, 0)$ has the following structure:

$$
\tilde{\mathcal{F}}^{(3)}(k, 0)=\left(\begin{array}{ccccccccc}
\tau_{n n} f_{n n} & \frac{\mathrm{i}}{k} f_{n n} & \tau_{n e} f_{n e} & 0 & f_{n e} & -\mathrm{i} k f_{J J} & 0 & 0 & -k \tilde{f}_{\dot{J} e} \\
\frac{\mathrm{i}}{k} f_{n n} & 0 & \frac{\mathrm{i}}{k} f_{n e} & f_{J J} & 0 & 0 & -\mathrm{i} \tilde{f}_{j e} & -f_{j j} & 0 \\
\tau_{n e} f_{n e} & \frac{\mathrm{i}}{k} f_{n e} & \tau_{e e} f_{e e} & 0 & f_{e e} & -\mathrm{i} \tilde{f}_{j e} & 0 & 0 & -f_{\dot{e} \dot{e}} \\
0 & -f_{J J} & 0 & 0 & \mathrm{i} \tilde{f}_{\dot{J} e} & f_{j j} & 0 & 0 & -\mathrm{i} \tilde{f}_{\ddot{J} \dot{e}} \\
-f_{n e} & 0 & -f_{e e} & \mathrm{i} \tilde{f}_{\dot{J} e} & 0 & 0 & f_{\dot{e} \dot{e}} & -\mathrm{i} \tilde{f}_{\ddot{J} \dot{e}} & 0 \\
-\mathrm{i} k f_{J J} & 0 & -\mathrm{i} \tilde{f}_{\dot{J} e} & -f_{\dot{J} j} & 0 & 0 & \mathrm{i} \tilde{f}_{\ddot{J} \dot{e}} & f_{\ddot{J} \ddot{J}} & 0 \\
0 & -\mathrm{i} \tilde{f}_{j e} & 0 & 0 & -f_{\dot{e} \dot{e}} & \mathrm{i} \tilde{f}_{\ddot{J} \dot{e}} & 0 & 0 & f_{\ddot{e} \ddot{e}} \\
0 & f_{j j} & 0 & 0 & -\mathrm{i} \tilde{f}_{\ddot{J} \dot{e}} & -f_{\ddot{J} \ddot{j}} & 0 & 0 & \mathrm{i} \tilde{f}_{\ddot{J} \ddot{e}} \\
k \tilde{f}_{j e} & 0 & f_{\dot{e} \dot{e}} & -\mathrm{i} \tilde{f}_{\ddot{J} \dot{e}} & 0 & 0 & -f_{\ddot{e} \ddot{e}} & \mathrm{i} \tilde{f}_{\ddot{J} \ddot{e}} & 0
\end{array}\right) .
$$

And the elements of matrix $\mathrm{L}^{(s)}(k)$ for $s=3$ [27],

$$
\mathrm{L}^{(3)}(k)=\left(\begin{array}{ccccccccc}
1 & 0 & 0 & 0 & 0 & 0 & 0 & 0 & 0 \\
0 & 1 & 0 & 0 & 0 & 0 & 0 & 0 & 0 \\
L_{e n} & 0 & 1 & 0 & 0 & 0 & 0 & 0 & 0 \\
\mathrm{i} L_{j n} & 0 & \mathrm{i} L_{j e} & 1 & 0 & 0 & 0 & 0 & 0 \\
0 & \mathrm{i} L_{\dot{e} J} & 0 & 0 & 1 & 0 & 0 & 0 & 0 \\
0 & L_{\ddot{J} J} & 0 & 0 & \mathrm{i} L_{\ddot{J} \dot{e}} & 1 & 0 & 0 & 0 \\
L_{\ddot{e} n} & 0 & L_{\ddot{e} e} & \mathrm{i} L_{\ddot{e} j} & 0 & 0 & 1 & 0 & 0 \\
\mathrm{i} L_{\ddot{J} n} & 0 & \mathrm{i} L_{\ddot{J} e} & L_{\ddot{J} j} & 0 & 0 & \mathrm{i} L_{\ddot{J} \ddot{e}} & 1 & 0 \\
0 & \mathrm{i} L_{\ddot{e} J} & 0 & 0 & L_{\ddot{e} \dot{e}} \mathrm{i} L_{\ddot{e} \ddot{J}} & 0 & 0 & 1
\end{array}\right),
$$

can be also expressed via static correlation functions, namely,

$$
\begin{aligned}
& L_{e n}=-\frac{f_{e n}}{f_{n n}}, \quad L_{j e}=-\frac{\tilde{f}_{j e}+k f_{J J} L_{e n}}{f_{e e}+L_{e n} f_{e n}}, \quad L_{j n}=-k \frac{f_{J J}}{f_{n n}}+L_{j e} L_{e n}, \\
& L_{\dot{e} J}=-\frac{\tilde{f}_{\dot{J} e}}{f_{J J}}, \quad L_{\ddot{J} \dot{e}}=-\frac{\tilde{f}_{\ddot{J} \dot{e}}+L_{\dot{e} J} f_{\dot{J} j}}{f_{\dot{e} \dot{e}}+L_{\dot{e} J} \tilde{f}_{\dot{J} e}}, \quad L_{\ddot{J} J}=\frac{f_{\dot{J} j}}{f_{J J}}-L_{\ddot{J} \dot{e}} L_{\dot{e} J}, \\
& L_{\ddot{e} j}=-\frac{\tilde{f}_{\ddot{j} \dot{e}}+L_{j e} f_{\dot{e} \dot{e}}+k L_{j_{n}} \tilde{f}_{j e}}{f_{j j}+L_{j e}^{2} f_{e e}+L_{j n}^{2} f_{n n}+2 L_{j e} L_{j_{n}} f_{e n}+2 L_{j e} \tilde{f}_{j_{e}}+2 k L_{j_{n}} f_{J J}}, \\
& L_{\ddot{e} e}=\frac{f_{\dot{e} e}+k L_{e n} \tilde{f}_{j e}}{f_{e e}+L_{e n} f_{e n}}-L_{\ddot{e} j} L_{j e}, \quad L_{\ddot{e} n}=k \frac{\tilde{f}_{\dot{j} e}}{f_{n n}}+\frac{f_{\dot{e} \dot{e}}+k L_{e n} \tilde{f}_{\dot{j} e}}{f_{e e}+L_{e n} f_{e n}} L_{e n}-L_{\ddot{e} j} L_{J_{n}},
\end{aligned}
$$




$$
\begin{aligned}
& L_{\tilde{J} \ddot{e}}=-\frac{F_{\ddot{J} Q}}{F_{Q Q}}, \quad L_{\ddot{J} j}=-L_{\ddot{J} \ddot{e}} L_{\ddot{e} j}+L_{\ddot{J} j}^{0}, \\
& L_{\ddot{J} e}=L_{\ddot{J} \ddot{e}} L_{\ddot{e} e}+L_{\tilde{J} j}^{0} L_{\dot{J} e}+\frac{\tilde{f}_{\ddot{J} \dot{e}}+k L_{e n} f_{j j}}{f_{e e}+L_{e n} f_{e n}}, \\
& L_{\ddot{J} n}=L_{\ddot{J} \ddot{e}} L_{\ddot{e} n}+L_{\ddot{J} j}^{0} L_{j_{n}}+\frac{\tilde{f}_{\ddot{J} \dot{e}}+k L_{e n} f_{\dot{J} j}}{f_{e e}+L_{e n} f_{e n}} L_{e n}+k \frac{f_{\dot{J} j}}{f_{n n}}, \\
& L_{\ddot{e} \ddot{J}}=-\frac{\tilde{f}_{\ddot{J} \ddot{e}}+L_{\ddot{J} \dot{e}} f_{\ddot{e} \ddot{e}}-L_{\ddot{J} J} \tilde{f}_{\ddot{J} \dot{e}}}{f_{\ddot{J} \ddot{J}}+L_{\ddot{J} \dot{e}}^{2} f_{\dot{e} \dot{e}}+L_{\ddot{J} J}^{2} f_{J J}-2 L_{\ddot{J} J} f_{j j}+2 L_{\ddot{J} \dot{e}} \tilde{f}_{\ddot{J} \dot{e}}-2 L_{\ddot{J} J} L_{\ddot{J} e} \tilde{f}_{j e}}, \\
& L_{\ddot{e} \dot{e}}=-L_{\ddot{e} \ddot{J}} L_{\ddot{J} \dot{e}}+L_{\ddot{e} \dot{e}}^{0}, \quad L_{\ddot{e} J}=L_{\ddot{e} \ddot{J}} L_{\ddot{J} J}+L_{\ddot{e} \dot{e}}^{0} L_{\dot{e} J}+\frac{\tilde{f}_{\ddot{J} \dot{e}}}{f_{J J}},
\end{aligned}
$$

where the following notations are used:

$$
\begin{aligned}
& F_{\ddot{J} Q}=\tilde{f}_{\ddot{J} \ddot{e}}+L_{\ddot{e} j} f_{\ddot{J} \ddot{J}}-L_{\ddot{e} e} \tilde{f}_{\ddot{J} \dot{e}}-k L_{\ddot{e} n} f_{\dot{J} \dot{J}}, \\
& F_{Q Q}=f_{\ddot{e} \ddot{e}}+L_{\ddot{e} j}^{2} f_{j j}+L_{\ddot{e} e}^{2} f_{e e}+L_{\ddot{e} n}^{2} f_{n n}-2 L_{\ddot{e} e} f_{\dot{e} e}-2 L_{\ddot{e} n} k \tilde{f}_{j e}+2 L_{\ddot{e} j} \tilde{f}_{\ddot{J} \dot{e}} \\
& -2 L_{\ddot{e} j} L_{\ddot{e} e} \tilde{f}_{J_{e}}+2 L_{\ddot{e} n} L_{\ddot{e} e} f_{e n}-2 L_{\ddot{e} j} L_{\ddot{e} n} f_{J J} \text {, } \\
& L_{\tilde{J} j}^{0}=\frac{f_{\ddot{J} j}+L_{\dot{J}_{e}} \tilde{f}_{\ddot{j} \dot{e}}+L_{j_{n}} k f_{j j}}{f_{j j}+L_{j e}^{2} f_{e e}+L_{J_{n}}^{2} f_{n n}+2 L_{j_{n}} L_{j e} f_{e n}+2 L_{j_{e}} \tilde{f}_{j_{e}}+2 k L_{j_{n}} f_{J J}}, \\
& L_{\ddot{e} \dot{e}}^{0}=\frac{f_{\dot{e} \dot{e}}+L_{\dot{e} J} \tilde{f}_{\ddot{J} \dot{e}}}{f_{\dot{e} \dot{e}}+L_{\dot{e} J}^{2} f_{J J}+2 L_{\dot{e} J} \tilde{f}_{\dot{J} e}} .
\end{aligned}
$$

Note that all the purely imaginary elements of matrix $L^{(s)}(k)$ are odd functions of $k$, and all the purely real elements of matrix $L^{(s)}(k)$ are even functions of $k$.

The recurrent relation $(2.21)$ for this case can be rewritten as follows:

$$
\begin{array}{r}
\tilde{\varphi}_{s-1}^{J J}(z)=\frac{\Gamma_{s-1}^{J J}}{z+\tilde{\varphi}_{s}^{J J}(z)-\frac{\left(-\mathrm{i} \Omega_{s}^{J h}+\tilde{\varphi}_{s}^{J h}(z)\right)\left(-\mathrm{i} \Omega_{s}^{h J}+\tilde{\varphi}_{s}^{h J}(z)\right)}{z+\tilde{\varphi}_{s}^{h h}(z)}}, \\
\tilde{\varphi}_{s-1}^{J h}(z)=\frac{\Gamma_{s-1}^{h h}}{-\mathrm{i} \Omega_{s}^{h J}+\tilde{\varphi}_{s}^{h J}(z)-\frac{\left(z+\tilde{\varphi}_{s}^{J J}(z)\right)\left(z+\tilde{\varphi}_{s}^{h h}(z)\right)}{-\mathrm{i} \Omega_{s}^{h J}+\tilde{\varphi}_{s}^{h J}(z)}}, \\
\tilde{\varphi}_{s-1}^{h J}(z)=\frac{\Gamma_{s-1}^{J J}}{-\mathrm{i} \Omega_{s}^{J h}+\tilde{\varphi}_{s}^{J h}(z)-\frac{\left(z+\tilde{\varphi}_{s}^{J J}(z)\right)\left(z+\tilde{\varphi}_{s}^{h h}(z)\right)}{-\mathrm{i} \Omega_{s}^{h J}+\tilde{\varphi}_{s}^{h J}(z)}} \\
\tilde{\varphi}_{s-1}^{h h}(z)=\frac{\Gamma_{s-1}^{h h}}{z+\tilde{\varphi}_{s}^{h h}(z)-\frac{\left(-\mathrm{i} \Omega_{s}^{h J}+\tilde{\varphi}_{s}^{h J}(z)\right)\left(-\mathrm{i} \Omega_{s}^{J h}+\tilde{\varphi}_{s}^{J h}(z)\right)}{z+\tilde{\varphi}_{s}^{J J}(z)}}
\end{array}
$$


where $s$ takes an arbitrary integer value $(s \geqslant 1)$ and " $h$ " denotes the dynamic variable $^{11}$ related to the energy density $\hat{e}_{k}$ (e.g., see [22]). The relations between the hydrodynamic memory functions and the memory functions of a five-variable description known previously [16], immediately follow from (3.13)-(3.16) when $s=1$. The hydrodynamic memory functions $(s=0)$ are directly related to the generalized transport coefficients by the expressions:

$$
\begin{array}{r}
\varphi_{J J}^{h, L}(k, z)=\frac{k^{2}}{n m} \tilde{\eta}^{(L)}(k, z)=\frac{k^{2}}{n m}\left[\tilde{\zeta}(k, z)+\frac{4}{3} \tilde{\eta}(k, z)\right], \\
\varphi_{J h}^{h}(k, z)=-\frac{\mathrm{i} k^{2}}{n C_{\mathrm{V}}(k) T} \tilde{\xi}(k, z), \quad \varphi_{h J}^{h}(k, z)=-\frac{\mathrm{i} k^{2}}{n m} \tilde{\xi}(k, z), \\
\varphi_{h h}^{h}(k, z)=\frac{k^{2}}{n C_{\mathrm{V}}(k)} \tilde{\lambda}(k, z), \quad \varphi_{J J}^{h, T}(k, z)=\frac{k^{2}}{n m} \tilde{\eta}(k, z),
\end{array}
$$

where $\tilde{\zeta}(k, z), \tilde{\eta}(k, z)$ and $\tilde{\lambda}(k, z)$ are the generalized bulk and shear viscosities, and the generalized thermal conductivity, respectively. The generalized coefficient $\tilde{\xi}(k, z)$ describes the dynamical coupling or cross correlation between the stress and heat currents which vanishes in the limit $k \rightarrow 0$ because of different properties of the variables $\hat{J}_{k}$ and $\hat{h}_{k}$ under the time reversal symmetry. For the same reason the imaginary unit $i$ is used in the definition of $\tilde{\xi}(k, z)$. It is evident that the expressions for $\tilde{\eta}(k, z), \tilde{\zeta}(k, z)$ and $\tilde{\lambda}(k, z)$, which follow from (3.17), reproduce the well-known Green-Kubo formulas $[1,2,4]$ in the hydrodynamic limit.

Hence, we have now all the expressions needed for a subsequent study of a simple fluid within the nine-mode approximation $(M=9)$. And it should be noted again that within this approach one can obtain the results for $M \times M$ time correlation functions, $M$ generalized collective modes, and all the generalized transport coefficients including the cross correlation coefficient $\tilde{\xi}(k, z)$, being usually neglected in common practice.

The results for the spectrum of generalized longitudinal collective modes, obtained $[22,24]$ for a Lennard-Jones fluid within the nine-mode description, are plotted in figure 2. From the first view these results could be treated as rather complicate for understanding. However, some important findings follow directly from our consideration. We see in figure 2 again that two types of generalized collective modes can be distinguished. Of all the eigenvalues, the damping coefficients of three eigenvalues vanish when $k$ tends to zero in full agreement with the hydrodynamic theory. They reduce to three hydrodynamic modes and have the same asymptotic behaviour when $k \rightarrow 0$, independently of $M$ (see also [22,23]). The three generalized hydrodynamic modes are two propagating sound modes denoted by " $s$ " in figure 2, with the eigenvalues $z_{\mathrm{s}}^{ \pm}(k)=\sigma_{\mathrm{s}}(k) \pm \mathrm{i} \omega_{\mathrm{s}}(k)$ for which in the hydrodynamic limit $k \rightarrow 0$ we have the well-known result $\sigma_{\mathrm{s}}(k) \simeq \Gamma k^{2}$ and $\omega_{\mathrm{s}}(k) \simeq c_{\mathrm{s}} k$, where $\Gamma$ is a sound-damping coefficient and $c_{\mathrm{s}}$ is the adiabatic velocity of sound as given by linear hydrodynamics, and a heat mode labelled by " $h$ ", with the purely real eigenvalue $z_{\mathrm{h}}(k)=\sigma_{\mathrm{h}}(k)$ for which in the hydrodynamic limit we

\footnotetext{
${ }^{11}$ The dynamic variable $\hat{h}_{k}$ describes the fluctuations of temperature (see, e.g., [3,32]).
} 

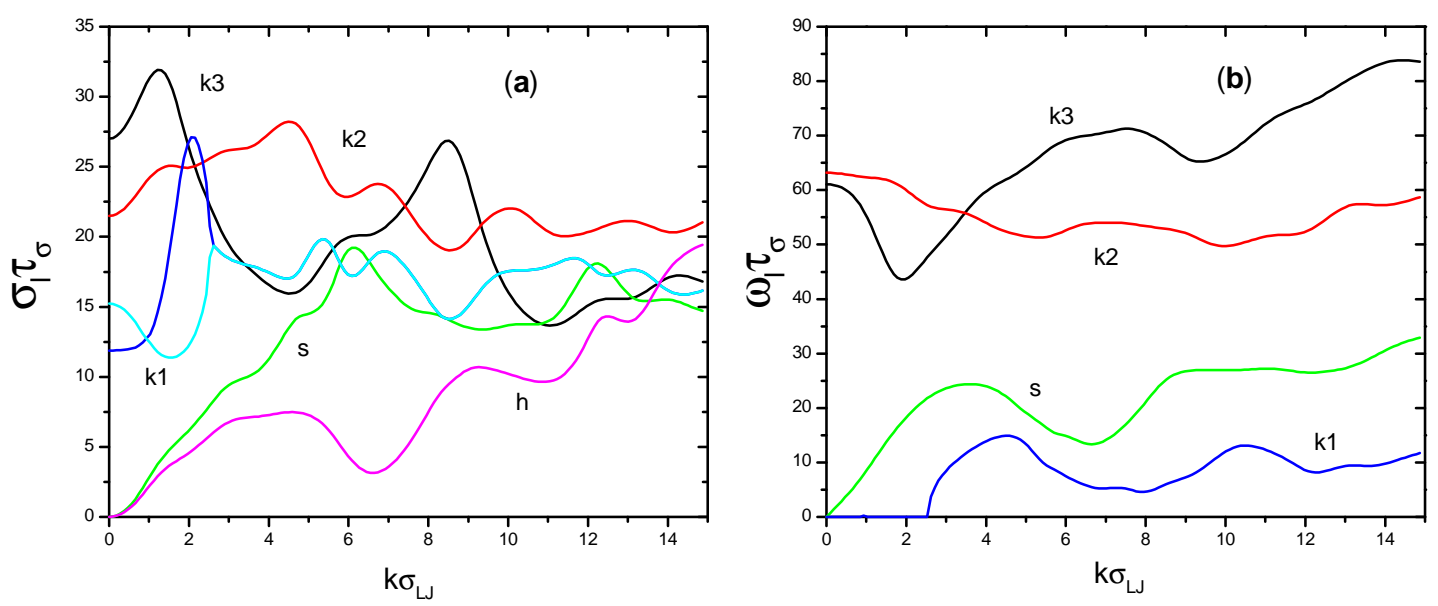

Figure 2. Generalized longitudinal mode spectrum of a LJ fluid in the nine-mode description at $n^{*}=0.845$ and $T^{*}=1.705$ : (a) damping coefficients and (b) dispersions. Note that two kinetic (k1) modes start as two purely relaxing modes and become complex-conjugated ones for $k \sigma_{\mathrm{LJ}}>2.5$. From [24].

have $\sigma_{\mathrm{h}}(k) \simeq D_{\mathrm{T}} k^{2}$, where $D_{\mathrm{T}}$ is a thermal diffusivity. The damping coefficients of all the other eigenvalues approach the finite values when $k$ goes to zero (see figure $2 \mathrm{a}$ ). They correspond to generalized kinetic modes, labelled by " $k_{\mathrm{N}}$ " in figure 2, where $\mathrm{N}$ is an integer number. For $M=9$ we found six kinetic modes which are complex-conjugated practically in the whole range of $k$ studied. There is only one exception for $k \sigma_{\mathrm{LJ}}<2.5$, where two purely relaxing kinetic modes exist. A similar result was also found [22] for $M=5$. As it had been expected, we observed a tendency of the results for the lowest-lying eigenvalues to converge with the increase of $M$. This was seen, in particular, for the generalized heat mode which was separated from other modes up to $k \sigma_{\mathrm{LJ}}<13$. Summing up all the features seen in figure 2 we note that:

(i) The generalized hydrodynamic modes are well separated from the others in the hydrodynamic limit, so that they have to contribute mainly to the time correlation functions when $k$ is small. Thus, the results of Navier-Stokes hydrodynamics are valid in the hydrodynamic limit.

(ii) When $5.0<k \sigma_{\mathrm{LJ}}<8.0$, the generalized heat mode is well separated from the others and a simplified one-mode description can be developed for the dynamic structure factor. That is the main reason why all the known dynamic models of fluids (e.g., hydrodynamic, viscoelastic, and thermoviscoelastic models) describe equally well the dynamic structure factor in the range of $k$ where the first maximum of the static structure factor is located.

(iii) There exist generalized kinetic modes (denoted by "k1", see figure 2b) which behave very similarly to the propagating shear modes found for the case of transverse fluctuations (compare with figure $1 \mathrm{~b}$ ). They are purely relaxing for $k \sigma_{\mathrm{LJ}}<2.5$ and become propagating ones for larger values of $k$. It can be shown that such a behaviour of these modes is the result of the dynamical coupling of viscoelastic and thermal processes, and they can be treated as propagating 
thermal excitations (like thermal waves [46]) transformed under the influence of viscoelastic properties. It is interesting to mention that in some particular cases generalized kinetic propagating excitations can be observed in experiments (see, e.g., $[48,49])$. In more detail the problem of propagating kinetic modes will be discussed elsewhere [29].

In figure 3 the dynamic structure factor $S(k, \omega)$ of the Lennard-Jones fluid is plotted as a function of frequency $\omega$ for six values of $k$, namely, $k=[1,3,7,10,15$, $20] k_{\min }$, where $k_{\min }=0.936 / \sigma_{\mathrm{LJ}}$. The MD data are shown as circles. The results of the nine-mode approximation ${ }^{12}$ are plotted by solid curves. The separated contributions from the kinetic, generalized hydrodynamic and generalized sound modes are also shown by dotted, dashed and long-dashed curves, respectively. One sees in figure 3 that the dynamic structure factor obtained within the nine-mode description is practically indistinguishable from the MD data for the entire region of $k$ studied herein. Note that for the dynamic structure factor in the nine-mode approximation $(s=3)$ the first nine frequency moments coincide with the true ones. Moreover, the value $S(k, 0)$ is also reproduced explicitly.

It is seen in figure 3 that:

(i) The relative contribution of the kinetic collective modes becomes more important when the value of $k$ increases. However, up to $k \sim 10 k_{\min }$ the main contribution to $S(k, \omega)$ is related to the generalized hydrodynamic modes as expected. It is due to an interplay of two different mechanisms of relaxations, namely, the hydrodynamic relaxation for smaller values of $k$ (say $k \sigma_{\mathrm{LJ}}<4.0$, see figure $2 \mathrm{~b}$ ), where all the hydrodynamic excitations are important, and the viscoelastic relaxation, which is determined mainly by the generalized heat mode, when $4.0<k \sigma_{\mathrm{LJ}}<8.0$. We see in figures $3 \mathrm{~b}, 3 \mathrm{c}$ and $3 \mathrm{~d}$ that the relative contribution of the generalized sound modes becomes negligible for $k$ where the principal maximum of $S(k)$ is located. Hence, an "effective" three-mode description could be used in a rather wide range of $k$, and it is in agreement with the conclusion of $[15,16]$. Effective parameters of such a simplified treatment can be found directly in the GCM approach as the parameters of the generalized hydrodynamic modes found in higher-order mode approximations.

(ii) The relative contributions from the kinetic and hydrodynamic terms are nearly the same for $k \sim 15 k_{\min }$, and then the kinetic contribution becomes dominant. In fact, the dynamic structure factor displays a Gaussian-like shape for $k \sim 20 k_{\min }$, and one can speak about the Gaussian-like behaviour herein. Nevertheless, one can see in figures $3 \mathrm{e}$ and $3 \mathrm{f}$ that the results obtained within the ninemode approximation are practically indistinguishable from the MD data. This is due to the fact that the sum rules are taken into account up to the eighth order including. Hence, one may conclude that the obtained results agree very well with the MD data for all the $k$ considered, starting from the hydrodynamic values and up to the Gaussian limit.

In figure 4 the reduced $k$-dependent generalized transport coefficients, namely,

\footnotetext{
${ }^{12}$ The results for $S(k, \omega)$ obtained in three-, five- and seven-mode approximations have been presented in $[22,23]$.
} 

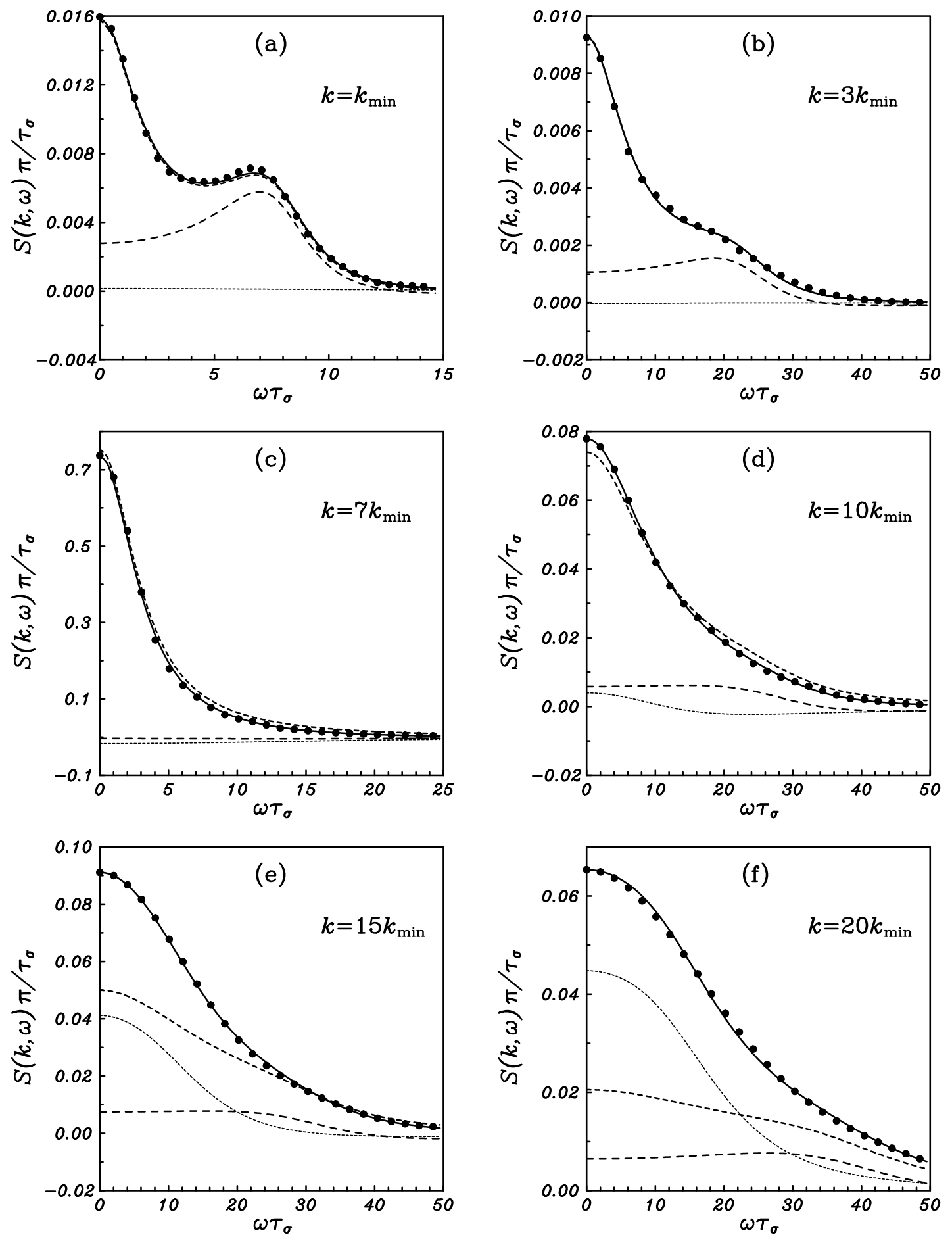

Figure 3. Dynamic structure factor of the LJ fluid at $n^{\star}=n \sigma_{\mathrm{LJ}}^{3}=0.845$ and $T^{\star}=k_{\mathrm{B}} T / \epsilon_{\mathrm{LJ}}=1.706$ as a function of $\omega \tau_{\sigma}$ for six values of $k \sigma_{\mathrm{LJ}}$ : (a) $k \sigma_{\mathrm{LJ}}=0.936$, (b) $k \sigma_{\mathrm{LJ}}=2.807$, (c) $k \sigma_{\mathrm{LJ}}=6.549$, (d) $k \sigma_{\mathrm{LJ}}=9.355$, (e) $k \sigma_{\mathrm{LJ}}=14.033$, (f) $k \sigma_{\mathrm{LJ}}=$ 18.710. The results of the nine-mode approximation are plotted by the solid curve. The separated contributions from the kinetic, generalized hydrodynamic and generalized sound modes are indicated by the dotted, dashed, and longdashed curves, respectively. From [26]. 
the generalized thermal conductivity $\bar{\lambda}(k)$ (figure $4 \mathrm{a}$ ),

$$
\bar{\lambda}(k)=\frac{\tau_{\sigma} \sigma_{\mathrm{LJ}}}{k_{\mathrm{B}}} \lambda(k)=\frac{\tau_{\sigma} \sigma_{\mathrm{LJ}}}{k_{\mathrm{B}}} \operatorname{Re} \tilde{\lambda}(k, z=0) ;
$$

the generalized shear viscosity $\bar{\eta}(k)$ (figure $4 \mathrm{~b}$ ),

$$
\bar{\eta}(k)=\frac{\tau_{\sigma} \sigma_{\mathrm{LJ}}}{m} \eta(k)=\frac{\tau_{\sigma} \sigma_{\mathrm{LJ}}}{m} \operatorname{Re} \tilde{\eta}(k, z=0) ;
$$

the generalized bulk viscosity $\bar{\zeta}(k)$ (figure $4 \mathrm{c}$ ),

$$
\bar{\zeta}(k)=\frac{\tau_{\sigma} \sigma_{\mathrm{LJ}}}{m} \zeta(k)=\frac{\tau_{\sigma} \sigma_{\mathrm{LJ}}}{m} \operatorname{Re} \tilde{\zeta}(k, z=0) ;
$$

and the coupling coefficient $\bar{\xi}(k)$ (figure $4 \mathrm{~d}$ ),

$$
\bar{\xi}(k)=\frac{\sigma_{\mathrm{LJ}}^{2}}{\epsilon_{\mathrm{LJ}}} \xi(k)=\frac{\sigma_{\mathrm{LJ}}^{2}}{\epsilon_{\mathrm{LJ}}} \operatorname{Re} \tilde{\xi}(k, z=0)
$$

are plotted as functions of $k \sigma_{\mathrm{LJ}}$ at $\omega=0\left(\tau_{\sigma}=\sigma_{\mathrm{LJ}} \sqrt{m / \epsilon_{\mathrm{LJ}}}\right)$. It is evident that in our approach the coefficients $\lambda(k), \eta(k), \zeta(k)$ and $\xi(k)$ can be considered as some kind of input parameters of the theory which directly follow from the generalized hydrodynamic consideration with $s=0$, and, as a consequence, they are independent of the order of approximation. However, we would like to discuss these results having in mind two main reasons. First, it should be noted that the simplest way for the calculation of $k$-dependent transport coefficients in MD simulations is to use the matrix relation (2.29) between the hydrodynamic memory functions (3.17) and the generalized hydrodynamic matrix $\mathcal{T}^{(0)}(k)$ defined on the initial hydrodynamic set $\hat{\mathbf{A}}_{0}(k)=\left\{\hat{n}_{k}, \hat{J}_{k}, \hat{e}_{k}\right\}$. So that, instead of the direct numerical evaluation of generalized transport coefficients, which is considerably more complicated in practice $^{13}$, simple matrix mathematics can be performed based on the results for static correlation functions, as well as for the correlation times (3.12), and all these quantities can be directly obtained in the MD experiment. And, second, little is known about the properties of generalized transport coefficients and the results are still open for interpretations. Let us discuss some of them.

Of special interest is the $k$-dependence of the cross correlation coefficient $\xi(k)$, which in common practice is considered as irrelevant and negligible. As it is seen in figure $4 \mathrm{~d}$, function $\xi(k)$ increases rapidly from zero to its maximal value at $k \sigma_{\mathrm{LJ}} \simeq 1.2$, when $k$ increases, and the maximal value of $\xi(k)$ is of about the same order of magnitude as the value of the bulk viscosity. The behaviour of $\xi(k)$, when $k$ tends to zero, is also a point for discussion. Because $k=0$ is not open to an inspection by $\mathrm{MD}$, we put $\xi(0)=0$ by hands ${ }^{14}$ in agreement with $[1-3,52-54]$.

\footnotetext{
${ }^{13}$ This is because these coefficients are expressed in terms of time-dependent correlation functions which involve much more complicated projected currents, as well as a much more complicated evolution operator.

${ }^{14}$ It should be mentioned that according to the prediction of the kinetic theory [52] one has $\xi(k) \sim k$, when $k$ is small. Another result follows from the mode coupling theory [53], namely, $\xi(k) \sim \sqrt{k}$.
} 

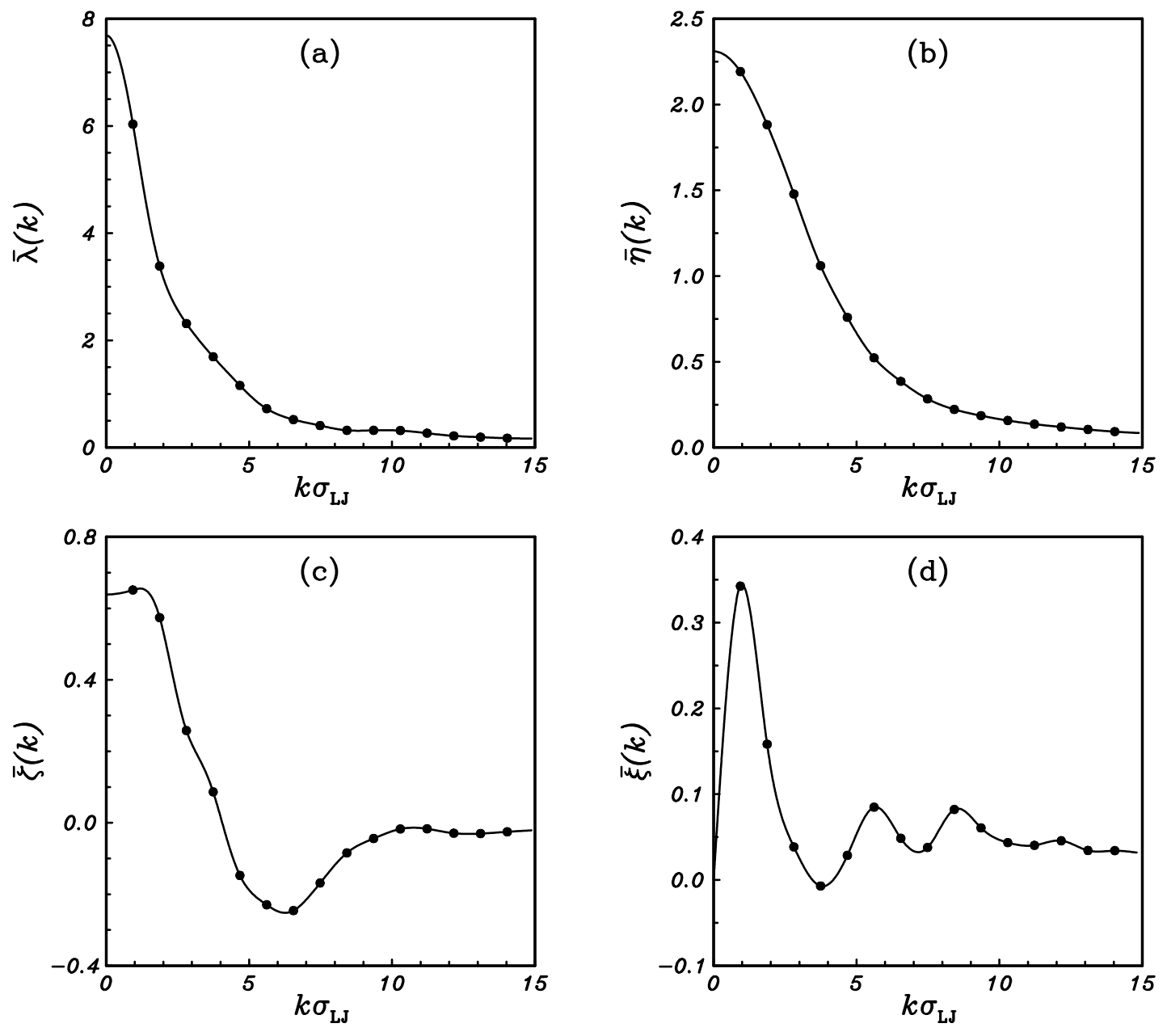

Figure 4. Reduced $k$-dependent transport coefficients of the LJ fluid at $n^{\star}=$ $n \sigma_{\mathrm{LJ}}^{3}=0.845$ and $T^{\star}=k_{\mathrm{B}} T / \epsilon_{\mathrm{LJ}}=1.706$, namely, the generalized thermal conductivity (a), the generalized shear (b) and the bulk (c) viscosities, and the cross correlation coefficient (d). From [27].

Thus, this coefficient can be important for intermediate and even small values of $k$, where the coupling of stress and heat fluxes becomes stronger. From this point of view we suppose that the meaning of the cross correlation coefficient was underestimated in the theory. For example, one may assume that the mode coupling effects (see, e.g., [53-55]) within the generalized hydrodynamics treatment can be correctly described by taking into account this dynamical coupling. To be more specific, sound dispersion for a Lennard-Jones fluid, obtained in the ninemode approximation, is plotted in figure 5. Comparing figures 5 and $4 \mathrm{~d}$, one may conclude that some sort of correlation is observed between a range of $k$, where the first maximum of $\xi(k)$ is located, and the values of $k$, for which $\omega_{\mathrm{s}}(k)$ is above $\omega_{\mathrm{s}}^{\text {hyd }}=c_{\mathrm{s}} k$. Other quite formal arguments might be also used, but it is beyond the scope of this paper. We note yet another example to show the role of the cross correlation coefficient in the hydrodynamic region. As it was mentioned in [3], it 


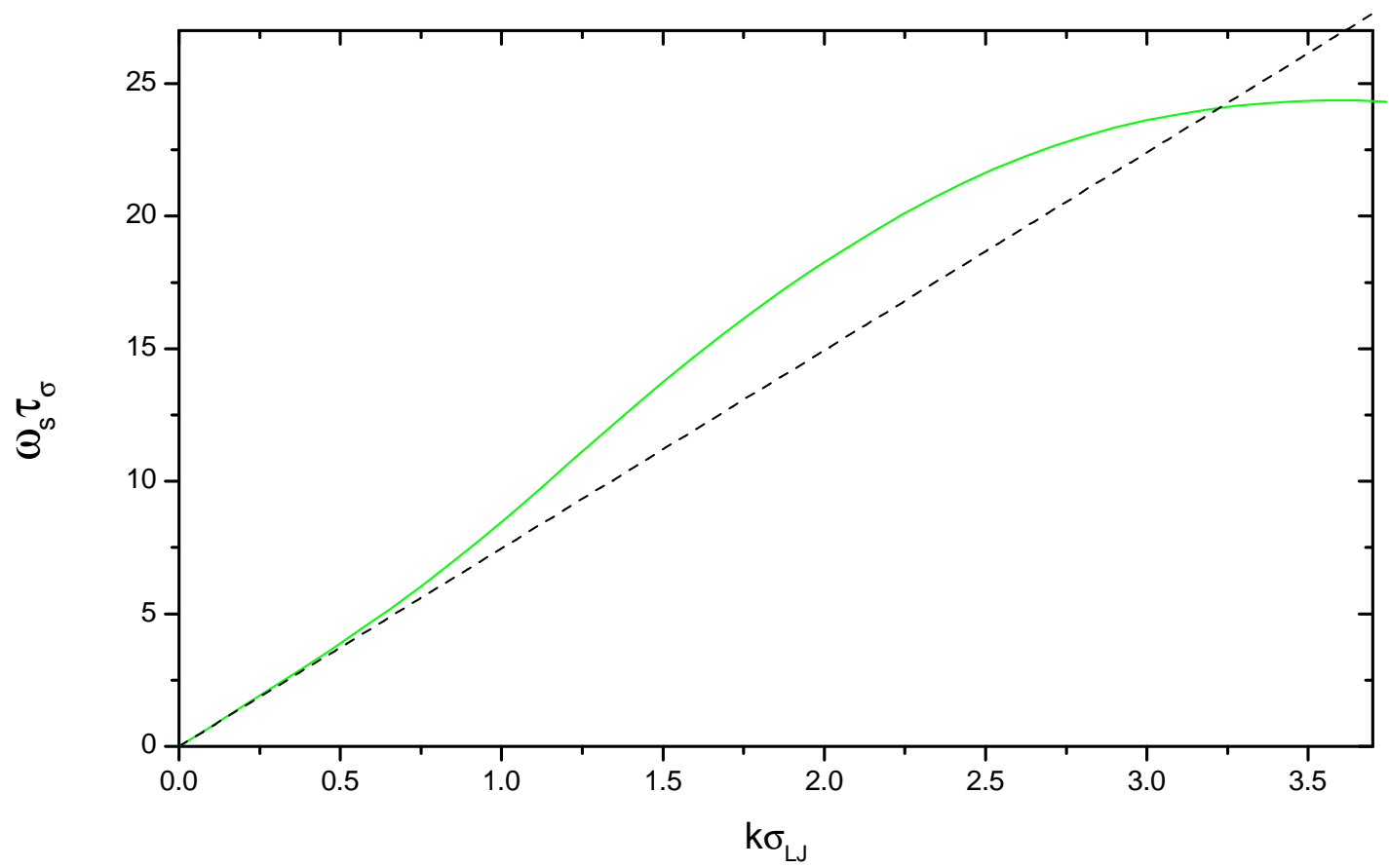

Figure 5. Sound dispersion of the LJ fluid at $n^{*}=0.845$ and $T^{*}=1.705$. The results of the nine-mode description are shown by the solid curve and the hydrodynamic dispersion $\omega_{\mathrm{s}}^{\text {hyd }}=c_{\mathrm{S}} k$ is plotted by the dashed line.

is evident from the MD data that function $S(k, \omega=0)$ is not well understood theoretically even for small values of $k$. Considering consistently an expansion for this function with respect to small parameter $k$, it can be seen [56] that the contribution from the ratio $\xi(k) / k$ appears in the second term together with the longitudinal viscosity coefficient.

The generalized transport coefficients for a dilute monoatomic gas have also been studied by Velasco and Garsia-Colin [52] within the Grad moment method. They used a somewhat different definition for the coupling coefficient $\xi(k)$ which was called in [52] a thermostriction coefficient and denoted by $\Gamma(k, \omega=0)$. The qualitative behaviour of $\xi(k)$ agrees with the result of [52].

Note in figure $4 \mathrm{c}$ that there exists a range of $k\left(k \sigma_{\mathrm{LJ}}>3.7\right)$ where the generalized bulk viscosity is negative. This is in agreement with the result obtained by Tankeshwar [57] for the Lennard-Jones fluid near the triple point.

Let us return to figure 5 and discuss the results obtained for $\omega_{\mathrm{s}}(k)$ within the GCM approach. One can see that $\omega_{\mathrm{S}}(k)$ is above $\omega_{\mathrm{S}}^{\text {hyd }}=c_{\mathrm{S}} k$ for $k \sigma_{\mathrm{LJ}}<3.3$ and tends to the hydrodynamic result $\omega_{\mathrm{s}}^{\text {hyd }}$, when $k$ decreases. This is in full agreement with the prediction of the mode coupling theory [53-55] for $\omega_{\mathrm{s}}(k)$, when $k$ is small. Namely, it was found that $\omega_{\mathrm{s}}(k)=c_{\mathrm{s}} k+a_{\mathrm{s}} k^{5 / 2}$ where $a_{\mathrm{s}}>0$. The value of $a_{\mathrm{s}}$ was estimated from a fitting procedure and correlates well with other results known in literature (see, e.g., [55]). Similar behaviour has also been found for the damping coefficients of the generalized sound and heat modes (see figure $2 \mathrm{a}$ ). We found that the generalized damping coefficients $\sigma_{\mathrm{s}}(k)$ and $\sigma_{\mathrm{h}}(k)$ are below their hydrodynamic 
asymptotics. Note that the mode coupling theory [53-55] predicts that $\sigma_{\mathrm{s}}(k)=$ $\Gamma k^{2}-a_{\mathrm{s}} k^{5 / 2}$ and $\sigma_{\mathrm{h}}(k)=D_{\mathrm{T}} k^{2}-a_{\mathrm{h}} k^{5 / 2}, a_{\mathrm{h}}>0$. So that one can say that the mode coupling effects are described within the GCM treatment at least on the qualitative level. To understand this relation between different theories an additional study has to be conducted.

The developed GCM scheme was used for the calculation of $(k, \omega)$-dependent transport coefficients in [27]. The numerical results obtained for the LennardJones fluid obviously show a tendency for their convergence when the order of mode approximations increases. The higher-order approximations (starting from the seven-variable one) describe well the $(k, \omega)$-dependence practically for all the wavenumbers and frequencies considered.

In [58], the free-parameter GCM approach was used for the study of the dynamic properties of liquid caesium near the melting point. The generalized thermodynamic quantities, the spectra of collective modes and the hydrodynamic time correlation functions were calculated in the five-variable description of longitudinal fluctuations. The obtained results showed reasonable agreement with the experimental data.

Other examples of the studies for monoatomic fluids within the GCM treatment could be given as well. For instance, very promising results were obtained for dense helium [48], an interesting development of the theory was proposed for liquid water [59], etc. However, let us turn our attention to possible applications of the GCM approach to the investigation of dynamic behaviour in more complicated model fluids. In the next section a brief overview of such applications will be presented.

\section{Other applications of the GCM approach}

\subsection{Binary mixtures}

During the last decade an essential progress in understanding the dynamical properties of binary systems was achieved [19-21,60]. The theoretical and experimental investigations showed that the dynamic structure factors $S(k, \omega)$ of liquid $\mathrm{Li}_{0.8}-\mathrm{Pb}_{0.2}$ [19], as well as of gas mixtures such as He-Ne [20,61] or He-Ar [21], display a behaviour which can be explained in terms of two additional pairs of propagating modes, namely, the so-called 'slow' and 'fast' sound modes. For very small $k$ the dynamic structure factor $S(k, \omega)$ of a mixture is described by the hydrodynamic Rayleigh-Brillouin quartet [62-64]: two Lorentzians centred at $\omega=0$ due to the concentration and heat diffusion and two Lorentzians located at $\omega= \pm \omega_{\mathrm{s}}(k)$ due to the hydrodynamic sound oscillations. Here $\omega_{\mathrm{s}}(k)=c_{\mathrm{s}} k$, where $c_{\mathrm{s}}$ is the adiabatic sound velocity of a mixture. However, it was found that the shape of $S(k, \omega)$ becomes essentially different for $k>k_{\mathrm{H}}$, with $k_{\mathrm{H}}$ being some limiting wavenumber for hydrodynamics. In particular, for $k>k_{\mathrm{H}}$, side peaks were also observed at frequencies $\pm \omega_{\mathrm{fs}}(k)$, with $\omega_{\mathrm{fs}}(k)>\omega_{\mathrm{s}}(k)$ due to the oscillations in the light component (the so-called 'fast sound') and at frequencies $\pm \omega_{\mathrm{ss}}(k)$, with $\omega_{\mathrm{ss}}(k)<\omega_{\mathrm{s}}(k)$ attributed to the oscillations of heavy particles (the so-called 'slow 
sound'). Hence, it is evident that these findings should be treated by methods of generalized hydrodynamics, and the known hydrodynamic results [62-64] are of little use in this case.

In [65], two propagating kinetic modes (i.e. different from the extended hydrodynamic sound) were determined for a hard-sphere fluid model of the mixtures with disparate masses within the revised Enskog theory, and the both pairs of propagating modes appeared at very small wavenumbers $k_{\mathrm{H}} \sim 0.005 \AA^{-1}$. Dispersion of one of them exhibited a phase velocity higher than that corresponding to the hydrodynamic sound (fast mode), and another evidenced a phase velocity below the sonic speed (slow mode).

A simple kinetic model, developed within the concept of GCM, was used for the description of a He-Ne mixture in [20]. In this model the dynamic structure factor $S(k, \omega)$ was presented as a sum of five Lorentzians, the parameters of which were calculated [20] using a fitting procedure for the experimental values of $S(k, \omega)$ of a dense gas mixture of $\mathrm{He}_{0.65}-\mathrm{Ne}_{0.35}$. So these results were obtained within the five-mode description and the model contained seven fitting parameters. In this study two propagating modes (fast and slow) were found, and it was predicted that the slow mode should merge with the hydrodynamic one for $k<k_{\mathrm{H}}$, with $k_{\mathrm{H}} \sim 0.007 \AA^{-1}$, whereas the fast mode vanishes in such a limit.

The problem of fast and slow excitations for dilute mixtures was considered for the so-called two-temperature model, (see [66] and references therein). In particular, it was predicted that a dilute mixture can show a propagating gap on the sound mode, depending upon the composition of the mixture, so that sound excitations can turn continuously into fast or slow propagating modes at large wavevectors.

Recently, the free-parameter GCM approach has been developed for the study of binary liquids [30,31]. For longitudinal fluctuations we have considered an enlarged set of dynamic variables containing, in addition to the densities of conserved quantities (the partial densities of particles $\hat{n}_{\alpha}(k)$ in $\alpha$ species, the total momentum density $\hat{J}(k)$, the density of the total energy $\hat{e}(k))$ and their time derivatives as well. It is easily seen [31] that taking into account the first order time derivatives $(s=1)$ one obtains a set of seven linear independent dynamic variables which determine the dynamics of binary systems in this approximation. In general, considering the approximation of the $s$ th order derivatives one deals with an $M$-mode description, where $M=4+3 s$. This is the first dissimilarity from the previous attempt [20] to extend the concept of GCM for binary mixtures. In [20], a five-variable description was used and the corresponding set of dynamic variables contained partial densities of particles $\hat{n}_{\alpha}(k)$, partial momentum densities $\hat{J}_{\alpha}(k)$, and the density of the total energy $\hat{e}(k)$. As it has been mentioned already, the choice of dynamic variables is of no significance when the explicit expressions for memory functions are used. And vice versa, applying the Markovian approximation for memory functions defined on a certain set of dynamic variables, we fix at the same time the physical processes to be considered, which can be more or less important for the purpose of our study. Thus, our main objective herein is to determine in a free-parameter scheme what is the minimal set of dynamic variables 
which correctly describe the appearance of 'fast sound' excitations.

In [30], the GCM approach, extended to binary mixtures in the free-parameter form, was applied to the study of the dynamical properties of a $\mathrm{He}_{0.65^{-}}$ $\mathrm{Ne}_{0.35}$ mixture. In particular, the spectra of the generalized collective modes were investigated for several various sets of dynamical variables, namely,

$$
\begin{gathered}
\hat{\mathbf{A}}^{\mathrm{H}}(\mathbf{k}, t)=\left\{\hat{n}_{1}(\mathbf{k}, t), \hat{n}_{2}(\mathbf{k}, t), \hat{\mathbf{J}}(\mathbf{k}, t), \hat{e}(\mathbf{k}, t)\right\}, \\
\hat{\mathbf{A}}^{5 \mathrm{~A}}(\mathbf{k}, t)=\left\{\hat{n}_{1}(\mathbf{k}, t), \hat{n}_{2}(\mathbf{k}, t), \hat{\mathbf{J}}_{1}(\mathbf{k}, t), \hat{\mathbf{J}}_{2}(\mathbf{k}, t), \hat{e}(\mathbf{k}, t)\right\}, \\
\hat{\mathbf{A}}^{5 \mathrm{~B}}(\mathbf{k}, t)=\left\{\hat{n}_{1}(\mathbf{k}, t), \hat{n}_{2}(\mathbf{k}, t), \hat{\mathbf{J}}(\mathbf{k}, t), \hat{e}_{1}(\mathbf{k}, t), \hat{e}_{2}(\mathbf{k}, t)\right\}, \\
\hat{\mathbf{A}}^{7 \mathrm{~A}}(\mathbf{k}, t)=\left\{\hat{n}_{1}(\mathbf{k}, t), \hat{n}_{2}(\mathbf{k}, t), \hat{\mathbf{J}}_{1}(\mathbf{k}, t), \hat{\mathbf{J}}_{2}(\mathbf{k}, t), \hat{e}(\mathbf{k}, t), \dot{\hat{\mathbf{J}}}(\mathbf{k}, t), \dot{\hat{e}}(\mathbf{k}, t)\right\}, \\
\hat{\mathbf{A}}^{7 \mathrm{~B}}(\mathbf{k}, t)=\left\{\hat{n}_{1}(\mathbf{k}, t), \hat{n}_{2}(\mathbf{k}, t), \hat{\mathbf{J}}(\mathbf{k}, t), \hat{e}_{1}(\mathbf{k}, t), \hat{e}_{2}(\mathbf{k}, t), \dot{\hat{\mathbf{J}}}(\mathbf{k}, t), \dot{\hat{e}}(\mathbf{k}, t)\right\} .
\end{gathered}
$$

The sets $\hat{\mathbf{A}}^{\mathrm{H}}(\mathbf{k}, t)$ and $\hat{\mathbf{A}}^{7 \mathrm{~A}}(\mathbf{k}, t)$ are a hydrodynamic set containing the densities of conserved quantities only and an extended hydrodynamic set $(s=1)$, respectively. The case of dynamic variables (4.2) was considered in [20]. Two additional cases with sets $\hat{\mathbf{A}}^{5 \mathrm{~B}}(\mathbf{k}, t)$ and $\hat{\mathbf{A}}^{7 \mathrm{~B}}(\mathbf{k}, t)$ may be treated as extensions of the hydrodynamic set (4.1), when the partial densities of energy are considered separately. These two last cases correspond to a two-temperature dynamic model of a binary mixture considered, for instance, in [66]. The spectra of GCM were calculated [30] for all the sets defined above. The propagating kinetic modes were found for three sets, namely, $\hat{\mathbf{A}}^{5 \mathrm{~A}}(\mathbf{k}, t), \hat{\mathbf{A}}^{7 \mathrm{~A}}(\mathbf{k}, t)$, and $\hat{\mathbf{A}}^{7 \mathrm{~B}}(\mathbf{k}, t)$. However, only for the extended set $\hat{\mathbf{A}}^{7 \mathrm{~A}}(\mathbf{k}, t)$, the propagating kinetic modes, which behave like 'fast sound' excitations, were determined. For other two cases the dispersions of propagating kinetic modes were smaller than the dispersion of the sound modes $\omega_{\mathrm{s}}(k)$ for all the considered values of $k$, so that they can be considered as 'slow sound' modes. Of course, taking some parameters as adjustable ones, one may change this property. One can assume that it was the case similar to the one found in [20] where the same binary mixture was studied.

Our results for the spectra of the generalized collective modes obtained for hydrodynamic and extended $(s=1)$ sets of dynamic variables are shown in figure 6 . The eigenvalues $z_{\alpha}$ in figures 6 are given in reduced units using the time scale $\tau_{\sigma}=\sqrt{\bar{m} \beta} / k_{\min }=3.109 \mathrm{ps}$, where $\bar{m}=c_{1} m_{1}+c_{2} m_{2}$ and $k_{\min }=0.1748 \AA^{-1}$. As it had been expected, for the hydrodynamic set (figures $6 \mathrm{a}$ and $6 \mathrm{~b}$ ) we found four generalized hydrodynamic modes which can be considered as an extension of usual hydrodynamic modes known in literature (see, e.g., $[63,64])$. In the hydrodynamic limit they are:

(i) two complex conjugated propagating modes which correspond to sound excitations with the eigenvalues

$$
z_{\mathrm{s}}^{ \pm}=\Gamma k^{2} \pm \mathrm{i} c_{\mathrm{s}} k
$$

where $c_{\mathrm{s}}$ and $\Gamma$ are the sound velocity and damping coefficient, respectively; 

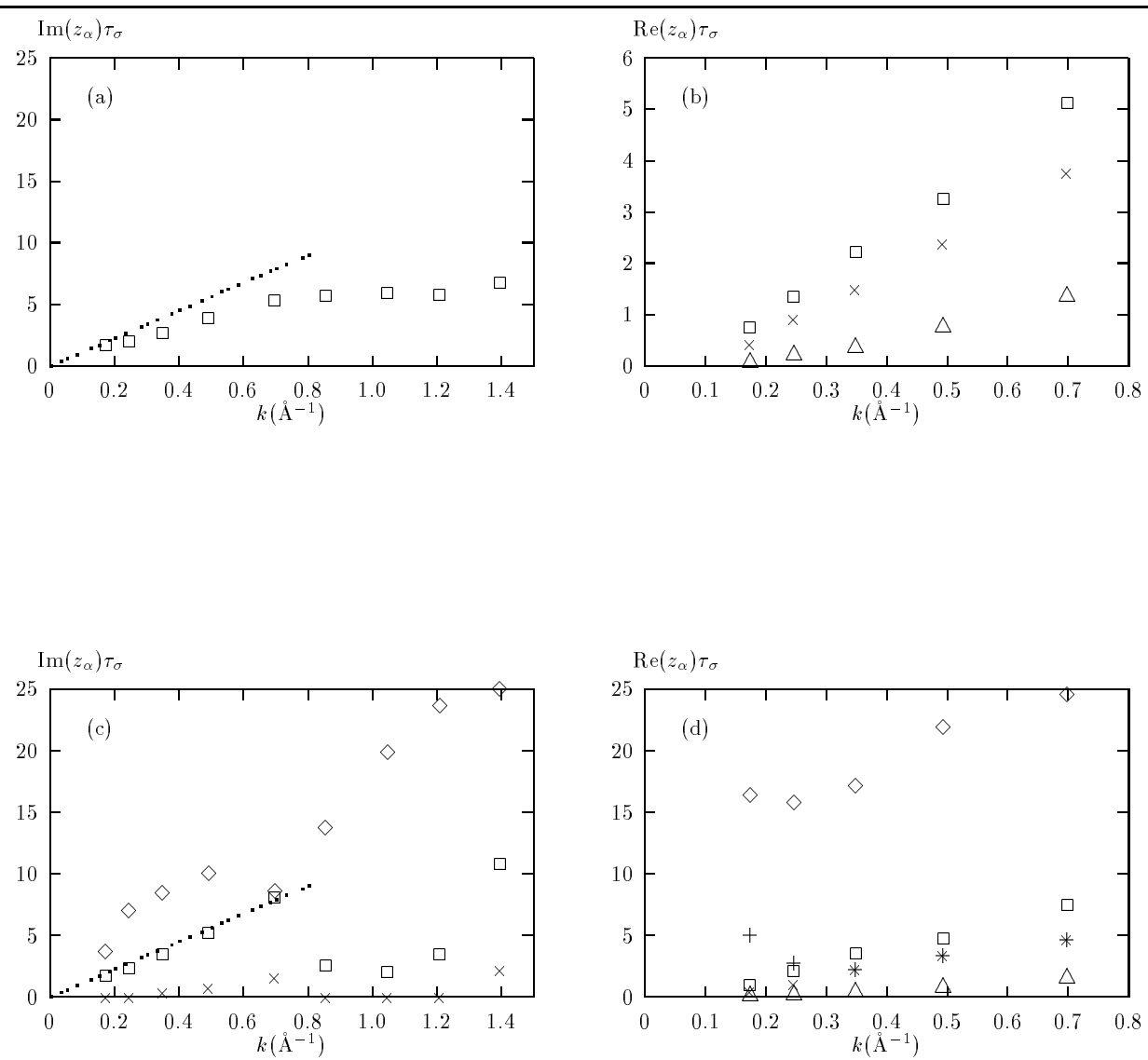

Figure 6. Generalized collective mode spectra obtained for hydrodynamic (a,b) and extended $(\mathrm{c}, \mathrm{d})$ sets of dynamic variables for a $\mathrm{He}_{0.65} \mathrm{Ne}_{0.35}$ mixture. The linear dispersion of sound with $c_{\mathrm{s}}=362 \mathrm{~m} / \mathrm{s}$ is plotted by a dotted line. The imaginary $(\mathrm{a}, \mathrm{c})$ and real $(\mathrm{b}, \mathrm{d})$ parts of the eigenvalues are shown for: sound modes by boxes; concentration modes by triangles; 'fast sound' modes by diamonds; 'slow sound' modes by $\times$. From [30].

(ii) two purely diffusive modes describing the concentration $(c)$ and heat $(h)$ fluctuations

$$
\begin{aligned}
& z_{\mathrm{c}}=D_{\mathrm{C}} k^{2}, \\
& z_{\mathrm{h}}=D_{\mathrm{T}} k^{2},
\end{aligned}
$$

with $D_{\mathrm{C}}$ and $D_{\mathrm{T}}$ being the concentration damping coefficient and thermal diffusivity, respectively.

The spectrum of the generalized collective modes calculated within the extended seven-variables set is shown in figures $6 \mathrm{c}$ and $6 \mathrm{~d}$. In this case we obtained seven eigenvalues $z_{\alpha}$. In the hydrodynamic limit we find (as it should be), in addition to the previous results (4.6)-(4.8), three kinetic modes $z_{k_{N}}(k)$ (denoted by crosses and diamonds in figure 6d) with finite damping coefficients,

$$
\lim _{k \rightarrow 0} \operatorname{Re} z_{k_{N}}(k)=z_{k_{N}}^{0}>0 .
$$

These kinetic modes describe the processes of a subsequent time scale which are faster in comparison with the hydrodynamic ones. 
In figure $6 \mathrm{~d}$, we can see that for larger values of $k$ there exists one purely real relaxing mode which may be considered an extended concentration mode (triangles ), and three pairs of complex conjugated propagating modes, one of which corresponds to the generalized sound excitations (labelled by boxes). Dispersion of the sound mode behaves as $c_{\mathrm{s}} k$ up to $k \simeq 0.7 \AA^{-1}$. Two other propagating modes vanish and reduce to the modes with purely real eigenvalues when $k$ decreases. We can see in figures $6 \mathrm{c}$ and $6 \mathrm{~d}$ that:

(i) For $k<0.25 \AA^{-1}$, the imaginary part of the eigenvalues for the propagating kinetic modes, denoted by $\times$ in figure $6 \mathrm{c}$, becomes zero and they degenerate for smaller values of $k$ into two relaxing modes (denoted by $\times$ and + in figure $6 \mathrm{~d}$ ), one of which is an extension of the heat mode. Note that the dispersion of these modes for $k>0.3 \AA^{-1}$ is always below $\omega_{\mathrm{s}}(k)$, so taking into account these properties and comparing them with MD data for the spectra of partial longitudinal currents we conclude that these lowest-lying propagating kinetic modes can be identified with 'slow sound' excitations;

(ii) The third pair of the propagating modes (plotted by diamonds) with the largest real part of the eigenvalues behaves in the range of small and intermediate wavenumbers $k$ quite similarly to the 'fast sound' modes found in [20]. This means that the dispersion of these modes, $\omega_{\mathrm{fs}}(k)$, is larger than the dispersion of the sound modes $\omega_{\mathrm{s}}(k)$. In our simulations, due to the system-size limitation we found $\omega_{\mathrm{fs}}(k)>\omega_{\mathrm{s}}(k)$ for all the considered values of $k$. However, there is an evident tendency that $\omega_{\mathrm{fs}}(k)$ tends to zero as $k$ goes to some fixed wavenumber $k_{\mathrm{H}}$ from above, so that for $k<k_{\mathrm{H}}$ these modes reduce to two relaxing kinetic modes with purely real eigenvalues. The value $k_{\mathrm{H}}$ can be defined by the equation $\omega_{\mathrm{fs}}\left(k_{\mathrm{H}}\right)=0$. From figure $6 \mathrm{c}$ one may estimate that $k_{\mathrm{H}} \sim 0.1 \AA^{-1}$. Due to the finite size of our MD ensemble (introducing $k_{\min }$ ) we were not able to give a more accurate result for $k_{\mathrm{H}}$. In general, these properties are in agreement with the conclusion given in [20] and the predictions of [65].

We also note that the results obtained for the propagating kinetic modes in a $\mathrm{He}_{0.65}-\mathrm{Ne}_{0.35}$ mixture within the free-parameter seven-variable description correlate very well with the locations of the side peaks in $S(k, \omega)$ measured by neutron scattering [20] and determined in MD simulations [61]. The generalized thermodynamic quantities, as well as the generalized transport coefficients for a $\mathrm{He}_{0.65}-\mathrm{Ne}_{0.35}$ mixture within the seven-mode approximation of the GCM approach have been calculated too $[30,68]$. A detailed analysis of contributions from the corresponding collective modes showed [30] that the 'fast sound' excitations in binary mixtures appear due to the dynamics of lighter particles, as it had been expected [65].

Summarizing, one may conclude that the 'fast sound' excitations observed $[19,20]$ in binary mixtures can be described within the GCM approach starting from the seven-mode approximation with an extended set of dynamic variables $\hat{\mathbf{A}}^{\mathrm{7A}}(k)$. Moreover, in this approximation all the three propagating modes, namely, extended sound, 'fast sound' and 'slow sound' appear. Now, one may recall once again that similar propagating kinetic modes were found for the Lennard-Jones fluid (see figure 2b, curve labelled by "k1"), but in that case the dispersion of 
propagating kinetic modes was below $\omega_{\mathrm{s}}(k)$ and these modes were not directly visible in the dynamic structure factor. However, it is pertinent to note that the 'fast sound' excitations were also observed in a monoatomic liquid, namely, in water at room temperature $[67,59]$. Moreover, in [59], the dispersions for the highfrequency propagating longitudinal and transverse modes were estimated by fitting the current-current correlation functions $C^{(l, t)}(k, \omega)$ calculated in MD simulations: in both cases the obtained results are very similar qualitatively to our results shown in figures $1 \mathrm{~b}, 2 \mathrm{~b}$, and $6 \mathrm{c}^{15}$. So, we conclude that the generalized propagating kinetic modes cannot be considered as a formal result of speculative mathematics, but they indeed have a real physical meaning and are observed in some cases experimentally.

In order to investigate the behaviour of 'fast sound' modes depending on mass ratio $m_{1} / m_{2}$, diameters ratio $\sigma_{1} / \sigma_{2}$ and the concentrations of particles $c_{1}$ and $c_{2}$ in the species we studied [69] the spectra of generalized longitudinal collective modes in He-Ar and Kr-Ar mixtures. The results have not been published yet, but the preliminary conclusions are the following:

(i) The width of the propagating gap for 'fast sound' excitations characterized by $k_{\mathrm{H}}$ becomes slightly narrow when the mass ratio increases (the case of a He-Ar mixture), so the range of a hydrodynamic description decreases.

(ii) For a Kr-Ar mixture where the mass ratio is not so large $\left(m_{\mathrm{Kr}} / m_{\mathrm{Ar}} \simeq 2\right.$, compare with $m_{\mathrm{Ne}} / m_{\mathrm{He}} \simeq 5$ and $m_{\mathrm{Ar}} / m_{\mathrm{He}} \simeq 9$ ), the propagating kinetic modes to be specified as 'fast sound' modes have not been found. In this case the behaviour of generalized collective modes is more similar to the result obtained for a simple fluid [24,58], where the higher-lying propagating kinetic modes were observed up to $k=0$.

The theoretical framework for the study of transverse fluctuations in a binary system is practically the same as in the case of a simple fluid (see Section 2.1). In [70] we applied the free-parameter generalized mode approach to the study of temperature dependence of transverse excitations in the glass-forming binary metallic alloy $\mathrm{Mg}_{70}-\mathrm{Zn}_{30}$ and found that the value of $k_{\mathrm{H}}$, where the propagating shear modes appear, decreases when the temperature decreases from the temperatures of a well-defined liquid state to the temperatures of an undercooled liquid. The value of $\eta(k=0)$ therewith rapidly increases and function $\eta(k)$ becomes narrower when the temperature decreases.

\subsection{Magnetic and polar fluids}

Yet another example where the generalized mode scheme can be applied is a magnetic liquid. Models of a magnetic liquid are of interest in the theory of magnetism because liquid magnets may have ideal soft-magnetic properties due to their isotropy. Starting from the 80-ies, thermodynamic, structural and dynamic properties of magnetic fluids were studied in many papers. The equilibrium be-

\footnotetext{
${ }^{15}$ In [59], an additional transverse optic-like mode was recognized. This mode may be treated as a higher-lying transverse propagating mode shown in figure $1 \mathrm{~b}$.
} 
haviour of the Heisenberg model ferrofluid was considered in [71,72]. The dynamic properties of liquid ferromagnets were studied mainly within phenomenological theories (see, e.g., [73,74]). However, some of the obtained results were somewhat contradictory. For example, one can note that the expressions for the sound velocity found within two main groups of phenomenological theories, belonging to the so-called 'co-rotational' and 'co-deformational' models of a ferrofluid, differ even qualitatively [74]. Hence, the problem arose to study the hydrodynamic behaviour by using a rigorous statistical treatment to start with.

In [75], with the help of the Zubarev method of a nonequilibrium statistical operator we derived generalized transport equations, equations for time correlation functions and a collective mode spectrum for a model of a magnetic fluid with isotropic interparticle interactions in the inhomogeneous external magnetic field. These equations were used for the subsequent investigation of hydrodynamic collective modes in [76]. In our study we have kept to the general outline described in this paper considering a set of conserved variables. It was found that the sound velocity of the Heisenberg-like model ferrofluid in the constant magnetic field is isotropic and can be expressed via an adiabatic compressibility at constant magnetization. In addition to the hydrodynamic sound and heat modes, the spin diffusion mode with a purely real eigenvalue was discovered. The microscopic expressions for the generalized thermodynamic quantities and generalized transport coefficients were derived as well. Using the explicit representation for the hydrodynamic matrix and applying the matrix perturbation theory, the analytic expressions for the dynamic structure factors of 'density-density' and 'spin density-spin density' were obtained [77] in the hydrodynamic limit. Note that all these results are directly applicable to the theory of polar liquids with isotropic interactions. As a consequence of the results obtained in [75-77], one has now a framework for the next study of generalized hydrodynamics of a magnetic fluid, and one of the most interesting problems here is the investigation of propagating spin waves in liquid magnets. Some aspects of this problem for solid magnets were considered in [28]. We note only that the spin waves in fluid magnets are, in fact, propagating kinetic modes which can be described in the similar manner as shear waves (see section 3.1).

In general, the problems of the microscopical study of the dynamical properties of magnetic fluids are also an interest of the theory of spin relaxation in liquids [78], as well as of the theory of orientational relaxation in ferrocolloidal fluids [79]. For our consideration this part is useful as an example that quantum effects can also be incorporated into the general scheme of the GCM approach discussed in this paper.

An interesting development of the free-parameter generalized mode approach was proposed in [80-82,50] for the description of the hydrodynamic and dielectric properties of dipolar fluids. The main idea consists in the fact that the microscopic operators of dipole density do not correlate at the basic level with molecular (orientationally-averaged) hydrodynamic densities of particles' number, spatial and angular momenta and total energy. For this reason, the dipole density and hy- 
drodynamic fluctuations can be considered relatively separately from each other, and it is no longer necessary to deal with the cumbersome hydrodynamic description [83] in which microscopic densities are orientationally-dependent. Therefore, to describe the processes of dielectric relaxation, an extended set of dynamical variables can be constructed on the dipolar-moment density and its first time derivatives $[81,82,50]$. The conserved quantities are not taken into account explicitly in such an approach, so that all the generalized collective modes appear to be kinetic ones. In fact, the proposed scheme can be considered as an extension of the three-variables theory of Madden and Kivelson [84], but a significant difference is that all the calculations were performed without any adjustable parameters. The collective mode spectra for longitudinal and transverse fluctuations of the dipole-moment density were evaluated for the Stockmayer fluid in a wide range of wavenumbers and it was shown that the dielectric permittivity can be reproduced quantitatively in the whole region of wavevectors and frequencies [50].

\subsection{Multicomponent mixture}

Until recently, the theoretical description of the dynamics for multicomponent fluids was based to a great extent on phenomenological approaches [85-88] and/or on the methods of the kinetic theory (see, e.g., $[89,90]$ ). The statistical derivation of hydrodynamic equations for a general mixture was given by Zubarev [37] with the help of a nonequilibrium statistical operator method. However, the expressions for generalized thermodynamic quantities, as well as for the generalized transport coefficients necessary for the generalized hydrodynamic treatment had not been available in literature. Hence, contrary to the cases considered above, our main purpose in this field was: to derive the basic equations for generalized hydrodynamics taking into account the nonlocal processes; to study these equations in the hydrodynamic limit of small wavenumbers and frequencies; to analyze the spectrum of the found hydrodynamic collective modes and to compare the obtained results with the results known in literature from phenomenological theories. Implementing this program we were able to create a framework for the next extension of the generalized mode approach for a multicomponent mixture.

In [91], following the line discussed above, we derived all the expressions needed for the subsequent study of a general mixture of chemically nonreactive fluids within the GCM approach. The obtained results may be summed up as follows:

(i) The microscopic set of the conserved (hydrodynamic) variables for longitudinal fluctuations in a multicomponent fluid can be introduced in the form:

$$
\hat{P}_{k}^{h}=\left\{\hat{\mathcal{N}}_{k}, \hat{J}_{k}, \hat{E}_{k}\right\}
$$

where $\hat{\mathcal{N}}_{k}$ is a column-vector, with the components $\hat{N}_{k, \alpha}$ being the number density of particles in $\alpha$ species; $\hat{J}_{k}$ is the density of the total current; and $\hat{E}_{k}$ is the total energy density. Starting from the set $\hat{P}_{k}^{h}$ and taking into account its time derivatives, one can apply the expressions derived in section 2 to the next consideration within the GCM approach. 
(ii) Using the theory of thermodynamic fluctuations, the expressions for generalized thermodynamic quantities via static correlation functions, constructed on the hydrodynamic variables (4.9), were derived. For instance, it was shown that the generalized compressibility $\kappa_{\mathrm{T}}(k)$ and the generalized partial molar volume $v_{\gamma}(k)$ in a multicomponent mixture could be defined as follows:

$$
\begin{gathered}
\frac{1}{\kappa_{\mathrm{T}}(k)}=n k_{\mathrm{B}} T \sum_{\alpha, \gamma=1}^{\nu} \frac{\sqrt{c_{\alpha} c_{\gamma}}}{S_{\alpha \gamma}(k)}, \\
v_{\gamma}(k)=\frac{k_{\mathrm{B}} T \kappa_{\mathrm{T}}(k)}{\sqrt{c_{\gamma}}} \sum_{\alpha=1}^{\nu} \frac{\sqrt{c_{\alpha}}}{S_{\alpha \gamma}(k)},
\end{gathered}
$$

where $S_{\alpha \gamma}(k)$ is a partial structure factor, so that the well-known thermodynamic relation

$$
\sum_{\alpha=1}^{\nu} c_{\alpha} v_{\alpha}=v=\frac{V}{N}
$$

is still valid in the generalized form, where $c_{\alpha}=N_{\alpha} / N$ is a concentration of particles in $\alpha$ species.

(iii) We have derived expressions for the generalized transport coefficients and extended for them some useful relations known in the hydrodynamic limit [37]. For example, for the generalized diffusion coefficients $\tilde{D}_{\alpha \beta}(k, z)$ and for the generalized thermal diffusion coefficients $\tilde{K}_{\alpha}(k, z)$ the following relations

$$
\sum_{\alpha=1}^{\nu} m_{\alpha} \tilde{D}_{\alpha \beta}(k, z)=\sum_{\beta=1}^{\nu} \tilde{D}_{\alpha \beta}(k, z) m_{\beta} \equiv 0
$$

and

$$
\sum_{\alpha=1}^{\nu} m_{\alpha} \tilde{K}_{\alpha}(k, z) \equiv 0
$$

are performed. Here $m_{\alpha}$ is the mass of particles in $\alpha$ species.

(iv) One to one correspondence has been demonstrated between the results for hydrodynamic collective modes obtained from the equations of generalized hydrodynamics and those found by phenomenological theories. Thus, we get two propagating complex-conjugated sound modes

$$
z_{\mathrm{s}}^{ \pm}= \pm c_{\mathrm{s}} k+\Gamma k^{2}
$$

and $\nu$ of purely real solutions describing a heat mode with

$$
z_{\mathrm{h}}=D_{\mathrm{T}} k^{2}
$$

as well as $(\nu-1)$ of diffusion modes with

$$
z_{d}^{r}=\mathcal{D}_{r} k^{2}, \quad r=1,2, \ldots, \nu-1
$$


where $D_{\mathrm{T}}$ and $\mathcal{D}_{r}$ are damping coefficients of the heat and diffusion modes, respectively. The explicit expressions for $c_{\mathrm{s}}, \Gamma, D_{\mathrm{T}}$ and $\mathcal{D}_{r}$ are given in [91].

Using the results of [91] and the general relations given in section 2, one can find the matrices $\mathcal{F}^{(s)}(k, 0), \tilde{\mathcal{F}}^{(s)}(k, 0)$, and $\mathrm{L}^{(s)}(k)$, defined on an extended set of dynamic variables, for a multicomponent fluid, so that the framework of the GCM study can be completed in such a way. This problem will be considered elsewhere. We note only that various phenomenological attempts to extend the hydrodynamic description for a multicomponent fluid by taking into account the non-hydrodynamic variables (such as partial currents, for instance) fit naturally into the GCM approach (see, e.g., [88]).

\section{Discussion and concluding remarks}

The really important changes in the study of fluid systems during the past decade were achieved in the way of the probing and interpretation of MD data and measurements by scattering experiments. Significant experimental advances were closely related to numerous attempts to bridge linearized hydrodynamics, the viscoelastic theory and the results of the kinetic theory. New experimental findings in this field (such as non-hydrodynamic behaviour of sound velocity in mixtures at small wavevectors $[92,93]$, collective excitations at intermediate or even large wavevectors in liquids [19-21,49], high-frequency phonon-like modes in simple fluids $[94,67,59]$, etc.) need their further interpretations and can be treated by appropriate methods which are, in fact, the methods of generalized hydrodynamics. In this paper one of the possible approaches to solving this problem is discussed, namely, the GCM approach. The results obtained for simple fluids and binary mixtures have evidenced that the dynamic properties of a fluid can be correctly described within the GCM approach in a wide range of wavevectors and frequencies, starting from the hydrodynamic values and up to the Gaussian-like limit. The consistent description of the both regions is indeed the main test for every theory to be considered a generalized hydrodynamic theory.

We finish our paper with a number of remarks and open questions.

(1) The main disadvantage of theories describing the dynamic behaviour at a fixed $(\mathbf{k}, \omega)$-range is the complexity of the used expressions, which makes it necessary either to use various theoretical approximations in their evaluation or to combine an analytical calculation with computer simulations. However, this general feature has a simple physical explanation: for fixed $k$ and $\omega$ one deals, in fact, with the properties of a mesoscopic system of particles which is macroscopically small but contains a large number of particles. This means that, on the one hand, the known thermodynamic relations should be modified taking into account the finite scale fluctuations at fixed $k$. On the other hand, for the intermediate time scale one has to involve into consideration many-particle-collision processes.

(2) As the result of generalization in thermodynamics, the so-called quasiequilibrium (or local equilibrium) statistical ensembles were introduced (see $[37,95]$ and references therein) where new variables, other than the locally conserved ones, 
can be raised into the status of independent variables. Thus, the idea of an extended set of variables appears already at this level of description.

(3) In $[96,52]$, the generalized hydrodynamic description was developed on the basis of the kinetic theory, starting directly from the Boltzmann equation by means of the Grad moment method. It was shown (see also [95]) that within the thirteenth moment approximation the single particle distribution function becomes not only a time dependent functional of the locally conserved densities, but of the heat flux and stress tensor as well. In the 26th moment approximation the theory becomes completely consistent with generalized hydrodynamics, and the corresponding relations between the generalized fluxes and the densities of conserved quantities known from hydrodynamics are then valid. Note that a fixed set of Grad moments can be considered as an extended set of dynamic variables taken into account.

(4) In fact, the mode coupling theories (see, e.g., [97,98]) are also based on extended sets of dynamic variables. The main difference between the cases discussed above and the mode coupling theories is the following: the mode coupling dynamic variables can be constructed in a form of orthogonalized products of conserved variables (see also [99]), such that each of the dynamic variables can be treated as an effective locally conserved quantity and nonlinear processes are included in the same manner. In the GCM approach the microscopic equations of motion for dynamic variables can also be written in the form of a local conservation law. For the densities of conserved quantities it is obvious and for other variables it follows from their specific definition. Nevertheless, we note that this might be a reason why the mode coupling effects are described (at least at the qualitative level) within the GCM approach in a higher mode approximation. This question remains to be clarified.

(5) A very promising idea was proposed by Zubarev et al. in [100], where a unified approach for the description of kinetic and hydrodynamic processes was developed $^{16}$. In this approach the set of dynamic variables contains, in addition to the densities of conserved hydrodynamic variables, the phase density of particles as well. The generalized transport equations were derived [100] in such a form that the kinetic equation for a nonorthogonal part of one-particle distribution function was coupled to the generalized hydrodynamic equations for averaged conserved quantities.

(6) The question $[4,15,16]$ whether the three exponentials description of dense fluids would be sufficient to describe the density-density time correlation function has been widely discussed in literature. In particular, it was shown that on the basis of three extended hydrodynamic modes the dynamic structure factor of a hard sphere [15] and Lennard-Jones fluids [16] can be fitted well. However, we note in this context that one has to consider another aspect of this problem, namely, whether the higher order sum rules could be taken into account within the description based on the extended hydrodynamic modes alone. The answer is obvious: the higher order sum rules cannot be reproduced correctly in such a case,

\footnotetext{
${ }^{16}$ In fact, it was the last work of Prof. D.N.Zubarev published after his death. It should be stressed that this paper was devoted to generalized hydrodynamics of fluids.
} 
and this means that the Gaussian-like behaviour in the limit of large wavenumbers cannot be well described.

(7) One of the most interesting problems of fluid dynamics, still actual, is to establish the meaning of the dynamic correlations which exist between a single particle and a collective behaviours. Some examples of such correlations are wellknown in literature. We recall only two of them, namely, the relation between the coherent dynamic structure factor $S(k, \omega)$ and the incoherent dynamic structure factor $S_{\mathrm{s}}(k, \omega)$ displaying for a large $k$ and the Stokes-Einstein like relation for the diffusion and shear viscosity coefficients. By itself, they imply the existence of some correlations between a single particle motion and the collective dynamics of particles, with both aspects being probed over essentially macroscopic space and time scales. While the theoretical interpretation of this correlation is surely footed [1-4], big challenges for the theory remain herein.

\section{Acknowledgements}

This work was prepared during my visit to the Institute for Theoretical Physics of Linz University. It is my pleasure to thank the Theory of Condensed Matter Group for their hospitality. I am indebted to Prof. M.Tokarchuk, Prof. R.Folk, Prof. V.Morozov, Prof. K.Kitahara, Dr. I.Omelyan, and Dr. T.Bryk for stimulating discussions.

Partial financial support was given by the Fonds für Förderung der wissenschaftlichen Forschung in Österreich (Austrian Science Foundation) under Project P 12422 TPH.

\section{References}

1. Boon J.P., Yip S. Molecular Hydrodynamics. New-York, McGraw-Hill Inc., 1980.

2. Hansen J.P., McDonald I.R. Theory of Simple Liquids. 2nd ed., London, Academic Press, 1986.

3. Copley J.R.D., Lovesey S.W. The dynamical properties of monoatomic liquids. // Rep. Prog. Phys., 1975, vol. 38, No. 4, p. 461-563.

4. Balucani U., Zoppi M. Dynamics of the Liquid State. Oxford, Clarendon Press, 1994.

5. Zwanzig R. Frequency-dependent transport coefficients in fluid mechanics. // J. Chem. Phys., 1965, vol. 43, No. 2, p. 714-720.

6. Mountain R.D., Zwanzig R. Shear relaxation times of simple fluids. // J. Chem. Phys., 1966, vol. 44, No. 7, p. 2777-2779.

7. Allain-Demoulin C., Lallemand P., Ostrowsky N. Theoretical study of light-scattering spectrum of a pure relaxing fluid: Application to viscous fluids at low temperature. // Mol. Phys., 1976, vol. 31, No. 2, p. 581-601.

8. Akcasu A.Z., Daniels E. Fluctuation analysis in simple fluids. // Phys. Rev. A, 1970, vol. 2, No. 3, p. 962-975.

9. Zubarev D.N., Tokarchuk M.V. Nonequilibrium statistical hydrodynamics of ionic systems. // Teor. Mat. Fiz., 1987, vol. 70, No. 2, p. 164-178. (in Russian).

10. Chung C.H., Yip S. Generalized hydrodynamics and time correlation functions. // Phys. Rev., 1969, vol. 182, No. 1 p. 323-338. 
11. Alley W.E., Alder D.J. Generalized transport coefficients for hard spheres. // Phys. Rev. A, 1983, vol. 27, No. 6, p. 3158-3173.

12. de Schepper I.M., Cohen E.G.D. Very-short-wavelength collective modes in fluids. // J. Stat. Phys., 1982, vol. 27, No. 2, p. 223-281.

13. Cohen E.G.D., de Schepper I.M., Zuilhof M.J. Kinetic theory of the eigenmodes of classical fluids and neutron scattering. // Physica B, 1984, vol. 127, p. 282-291.

14. Bruin C., Michels J.P.J., van Rijs J.C., de Graaf L.A., de Schepper I.M. Extended hydrodynamic modes in a dense hard sphere fluid. // Phys. Lett. A, 1985, vol. 110, No. 1 , p. 40-43.

15. Kamgar-Parsi B., Cohen E.G.D., de Schepper I.M. Dynamic processes in hard-sphere fluids. // Phys. Rev. A, 1987, vol. 35, No. 11, p. 4781-4795.

16. de Schepper I.M., Cohen E.G.D., Bruin C., van Rijs J.C., Montrooij W., Graaf L.A. Hydrodynamic time correlation functions for Lennard-Jones fluids. // Phys. Rev. A, 1988, vol. 38 , No. 1, p. 271-287.

17. Cohen E.G.D., de Schepper I.M. Effective eigenmode description of dynamic processes in dense classical fluid mixtures. // Nouvo Cimento D, 1990, vol. 12, No. 4/5, p. $521-542$.

18. Bertolini D., Tani A. Generalized hydrodynamics and the acoustic modes of water: Theory and simulation results. // Phys. Rev. E, 1995, vol. 51, No. 2, p. 1091-1118.

19. Bosse J., Jacucci G., Ronchetti M., Schirmacher W. Fast sound in two-component liquids. // Phys. Rev. Lett., 1986, vol. 57, No. 26, p. 3277-3279.

20. Westerhuijs P., Montfrooij W., de Graaf L.A., de Schepper I.M. Fast and slow sound in a dense gas mixture of helium and neon. // Phys. Rev. A, 1992, vol. 45, No. 6, p. $3749-3762$.

21. Smorenburg H.E., Crevecoeur R.M., de Schepper I.M. Fast sound in a dense helium argon gas mixture. // Phys. Lett. A, 1996, vol. 211, p. 118-124.

22. Mryglod I.M., Omelyan I.P., Tokarchuk M.V. Generalized collective modes for the Lennard-Jones fluid. // Mol. Phys., 1995, vol. 84, No. 2, p. 235-259.

23. Omelyan I.P., Mryglod I.M. Generalized collective modes of a Lennard-Jones fluid. High-mode approximation. // Cond. Matt. Phys. (Ukraine), 1994, vol. 4, p. 128-160.

24. Mryglod I.M., Omelyan I.P. Generalized collective modes for a Lennard-Jones fluid in higher mode approximations. // Phys. Lett. A, 1995, vol. 205, p. 401-406.

25. Mryglod I.M., Omelyan I.P. Generalized mode approach: 1. Transverse time correlation functions and generalized shear viscosity of a Lennard-Jones fluid. // Mol. Phys., 1997, vol. 90, No. 1, p. 91-99.

26. Mryglod I.M., Omelyan I.P. Generalized mode approach: 2. Longitudinal time correlation functions of a Lennard-Jones fluid. // Mol. Phys., 1997, vol. 91, No. 6, p. $1005-1015$.

27. Mryglod I.M., Omelyan I.P. Generalized mode approach: 3. Generalized transport coefficients of a Lennard-Jones fluid. // Mol. Phys., 1997, vol. 92, No. 5, p. 913-927.

28. Kalashnikov V.P., Tretyakov S.V. // Fiz. Metal. i Metallov., 1977, vol. 44, p. 710 (in Russian).

29. Mryglod I.M. Propagating kinetic modes in fluids. // J. Phys. Studies (Ukraine), 1998 (in preparation).

30. Bryk T.M., Mryglod I.M., Kahl G. Generalized collective modes in a binary $\mathrm{He}_{0.65^{-}}$ $\mathrm{Ne}_{0.35}$ mixture. // Phys. Rev. E, 1997, vol. 56, No. 3, p. 2903-2915.

31. Mryglod I.M., Ignatyuk V.V. Generalized hydrodynamics of binary mixtures. // 
J. Phys. Studies (Ukraine), 1997, vol. 1, No. 2, p. 181-190 (in Ukrainian).

32. Schofield P. Wavelength-dependent fluctuations in classical fluids: 1 . The long wavelength limit. // Proc. Phys. Soc., 1966, vol. 88, p. 149-170.

33. Zwanzig R. Lectures in Theoretical Physics. Eds. W.E.Britton, B.W.Downs, J.Downs, Vol.III, New York, Interscience, 1961.

34. Mori H. Transport, collective motion, and Brownian motion. // Prog. Theor. Phys., 1965, vol. 33, No. 3, p. 423-455.

35. Mori H. A continued-fraction representation of the time correlation functions. // Prog. Theor. Phys., 1965, vol. 34, No. 3, p. 399-416.

36. Zubarev D.N. Modern methods of the statistical theory of nonequilibrium processes.In: Itogi Nauki i Tekhniki, Sovr. Prob. Mat./ VINITI, 1980, vol. 15, p. 131-226 (in Russian).

37. Zubarev D.N. Nonequilibrium Statical Thermodynamics. New-York, Consultant Bureau, 1974.

38. Zubarev D., Morozov V., Röpke G. Statistical Mechanics of Nonequilibrium Processes: 1. Basic Concepts, Kinetic Theory. Berlin, Academie Verlag, 1996.

39. Zubarev D., Morozov V., Röpke G. Statistical Mechanics of Nonequilibrium Processes: 2. Relaxation and Hydrodynamic Processes. Berlin, Academie Verlag, 1997.

40. Mryglod I.M., Hachkevych A.M. On non-equilibrium statistical theory of a fluid: Linear relaxation theories. // Cond. Matt. Phys., 1995, vol. 5, p. 105-118.

41. Mryglod I.M., Hachkevych A.M. On non-equilibrium statistical theory of simple fluids: The basic equations. Preprint of the Institute for Condensed Matter Physics, ICMP-96-2E, Lviv, 1996, 22 p.

42. Mryglod I.M., Hachkevych A.M. Generalized hydrodynamics of fluids: I. Conception of generalized collective modes. // Ukr. Fiz. Zhurn., 1997, vol. 42, No. 4, p. 423-430 (in Ukrainian).

43. Mryglod I.M. Generalized hydrodynamics of fluids: II. Time correlation functions in generalized collective mode approach. // Ukr. Fiz. Zhurn., 1998, vol. 43, No. 2, p. 252-256 (in Ukrainian).

44. Bansal R., Pathak K.N. Sum rules and atomic correlations in classical liquids. // Phys. Rev. A, 1974, vol. 9, No. 6, p. 2773-2782.

45. Mryglod I.M., Hachkevych A.M. Approximate calculation of the memory functions and "shoulder" problem for a generalized shear viscosity. Preprint of the Institute for Condensed Matter Physics, ICMP-98-2U, Lviv, 1998, 19 p. (in Ukrainian).

46. Joseph D.D. Heat waves. // Rev. Mod. Phys., 1989, vol. 61, No. 1, p. 41-73.

47. Balucani U., Vallauri R., Gaskell T. Transverse current and generalized shear viscosity in liquid rubidium. // Phys. Rev. A, 1987, vol. 35, No. 10, p. 4263-4272.

48. Montfrooij W., de Graaf L.A., de Schepper I.M. Propagating microscopic temperature fluctuations in a dense helium fluid at 13.3 K. // Phys. Rev. B, 1992, vol. 45, No. 6, p. 3111-3114.

49. Teixeira J., Bellissent-Funel M.C., Chen S.H., Dorner B. Observation of new shortwavelength collective excitations in heavy water by coherent inelastic neutron scattering. // Phys. Rev. Lett., 1985, vol. 54, No. 25, p. 2681-2683.

50. Omelyan I.P., Mryglod I.M., Tokarchuk M.V. Generalized dipolar modes of a Stockmayer fluid in high-order approximations. // Phys. Rev. E, 1998, vol. 57, No. 6, p. $6667-6676$.

51. Bryk T.M., Mryglod I.M. Transverse excitations and generalized shear viscosity in 
liquid Cs near the melting point. // J. Phys. Studies, 1998, vol. 2, No. 3, p.322-330.

52. Velasco R.M., Garsia-Colin L.S. Generalized hydrodynamics in gases. // J. NonEquilib. Thermodyn., 1995, vol. 20, No. 1, p. 1-18.

53. Morozov V.G. Nonlocal hydrodynamics and dispersion of transport coefficients in simple fluid. // Physica A, 1983, vol. 117, No. 3-4, p. 511-530.

54. Ernst M.H., Dorfman J.R. Monoatomic dispersion relation for classical fluids. II. The general fluids. // J. Stat. Phys., 1975, vol. 12, No. 4, p. 311-359.

55. de Schepper I.M., Verkerk P., van Well A.A., de Graaf L.A. Non-analytic dispersion relations in liquid argon. // Phys. Lett. A, vol. 104, No. 1, p. 29-32.

56. Mryglod I.M., Ignatyuk V.V., Hachkevych A.M., 1998 (unpublished).

57. Tankeshwar K. Generalized negative bulk viscosity in liquids. // J. Phys.: Cond. Matt., 1994, vol. 6, p. 9295-9300.

58. Bryk T., Chushak Ya. Generalized collective modes in liquid Cs near melting point. // J. Phys.: Cond. Matt., 1997, vol. 9, p. 3329-3341.

59. Balucani U., Brodholt J.P., Vallauri R. Dynamical properties of liquid water. // J. Phys.: Cond. Matt., 1996, vol. 8, p. 9269-9274.

60. Smorenburg H. Microscopic Dynamics of Binary Mixtures and Quasi-colloidal Systems. Ph.D. Thesis, Delft, TU Delft, 1996.

61. Enciso E., Almarza N.G., Dominguez P., González M.A., Bermejo F.J. Dynamic structure factor of a helium-neon dense gas mixture: Crossover from hydrodynamics to the microscopic regime. // Phys. Rev. Lett., 1995, vol. 74, No. 21, p. 4233-4236.

62. March N.M., Tosi M.P. Atomic Dynamics in Liquids. London, Macmillan, 1976.

63. Bhatia A.B., Thornton D.E., March N.H. Dynamic structure factors for a fluid binary mixture in the hydrodynamic limit. // Phys. Chem. Liq., 1974, vol. 4, p. 97-111.

64. Mountain R.D., Deutch J.M. Light scattering from binary solutions. // J. Chem. Phys., 1969, vol. 50, No. 3, p. 1103-1108.

65. Campa A., Cohen E.G.D. Observable fast kinetic eigenmode in binary noble-gas mixtures? // Phys. Rev. Lett., 1988, vol. 61, No. 7, p. 853-856.

66. Schram R.P.C., Wegdam G.H. Fast and slow sound in two-temperature model. // Physica A, 1994, vol. 203, No. 1, p. 33-52.

67. Balucani U., Ruocco G., Torcini A., Vallauri R. Fast sound in liquid water. // Phys. Rev. E, 1993, vol. 47, No. 3, p. 1677-1684.

68. Mryglod I.M., Bryk T.M., Ignatyuk V.V. Generalized transport coefficients of a binary mixture. // Mol. Phys., 1998 (in preparation).

69. Bryk T.M., Mryglod I.M., 1998 (unpublished).

70. Bryk T.M., Mryglod I.M. Spectra of transverse excitations in glass-forming metallic alloy $\mathrm{Mg}_{70} \mathrm{Zn}_{30}$ : Temperature dependence. // Cond. Matt. Phys., 1999 (in press).

71. Vakarchuk I.A., Ponedilok G.V., Rudavskii Yu.K. Theory of liquid magnets. // Teor. Mat. Fiz., 1984, vol. 58, p. 291-302 (in Russian).

72. Vakarchuk I.A., Rudavskii Yu.K., Ponedilok G.V. Free energy of the amorphous ferromagnets with Heisenberg exchange Interaction and liquid-like disorder. // Phys. Stat. Sol. B, 1985, vol. 128, p. 231-242.

73. Akhiezer I.A., Akhiezer I.T. Oscillations of a ferromagnet liquid. // Sov. Phys. JETP, 1984, vol. 59, No. 1, p. 68-70.

74. Hubbard J.B., Stiles P.J. Hydrodynamics of magnetic and dielectric colloidal systems. // J. Chem. Phys., 1986, vol. 84, No. 12, p. 6955-6968.

75. Mryglod I.M., Tokarchuk M.V., Folk R. On the hydrodynamic theory of a magnetic 
liquid. I. General description. // Physica A, 1995, vol. 220, No. 3-4, p. 325-348.

76. Mryglod I.M., Folk R. On the hydrodynamic theory of a magnetic liquid. II. Hydrodynamic modes in the Heisenberg fluid. // Physica A, 1996, vol. 234, No. 1-2, p. $129-150$.

77. Mryglod I.M., Folk R., Dubyk S., Rudavskii Yu. Dynamic structure factors of a Heisenberg model ferrofluid. // Cond. Matt. Phys., 1999 (in press).

78. Electron Spin Relaxation in Liquids. Eds. L.T.Muus and P.W.Atkins, New York London, Plenum Press, 1972.

79. Rosensweig R.E. Ferrohydrodynamics. Cambridge, Cambridge Univ. Press, 1985.

80. Omelyan I.P., Zhelem R.I., Tokarchuk M.V. // Ukr. Fiz. Zhurn., 1997, vol. 42, p. 684 (in Ukrainian).

81. Omelyan I.P. Generalized collective mode approach in the dielectric theory of dipolar systems. // Physica A, 1997, vol. 247, No. 1-4, p. 121-139.

82. Omelyan I.P., Mryglod I.M., Tokarchuk M.V. Dielectric relaxation in dipolar fluid. Generalized mode approach. // Cond. Matt. Phys., 1998, vol. 1, No. 1(13), p.179-200.

83. Bagchi B., Chandra A. // Adv. Chem. Phys., 1991, vol. 80, p. 1.

84. Madden P., Kivelson D. // Adv. Chem. Phys., 1984, vol. 56, p. 467.

85. de Groot S.P., Mazur P. Non-equilibrium Thermodynamics. New York, Dover Publ., 1984.

86. Landau L.D., Lifschitz E.M. Fluid Mechanics. London, Pergamon Press, 1955.

87. Jordan P.C., Jordan J.R. Nonequilibrium statistical mechanics of a multicomponent fluid. // J. Chem. Phys., 1966, vol. 45, No. 7, p. 2492-2507.

88. Miyazaki K., Kitahara K., Bedeaux D. Nonequilibrium thermodynamics of multicomponent systems. // Physica A, 1995, vol. 230, No. 3-4, p. 600-630.

89. Boley C.D., Yip S. Kinetic theory of time-correlation functions in a binary gas mixture. // Phys. Fluids, 1972, vol. 15, No. 8, p. 1433-1446.

90. Lorez de Haro M., Cohen E.G.D. The Enskog theory for multicomponent mixtures. III. Transport properties of dense binary mixtures with one tracer component. // J. Chem. Phys., 1984, vol. 80, No. 1, p. 408-415.

91. Mryglod I.M. Generalized hydrodynamics of multicomponent fluids. // Cond. Matt. Phys., 1997, vol. 10, p. 115-135.

92. Wegdam G.H., Bot A., Schram R.P.C., Schaink H.M. Observation of fast sound in disparate-mass gas mixtures by light scattering. // Phys. Rev. Lett., 1989, vol. 63, No. 25, p. 2697-2700.

93. Campa A., Cohen E.G.D. Kinetic-sound propagation in dilute gas mixture. // Phys. Rev. A, 1989, vol. 39, No. 9, p. 4909-4911.

94. Sette F., Ruocco G., Krisch M., Masciovecchio C., Verbeni R., Bergmann U. Transition from normal to fast sound on liquid water. // Phys. Rev. Lett., 1996, vol. 77, No. 1 , p. $83-86$.

95. Garsia-Colin L.S. Extended irreversible thermodynamics: an unfinished task. // Mol. Phys., 1995, vol. 86, No. 4, p. 697-706.

96. Balabanyan G.O. Construction of generalized hydrodynamic asymptotics for classical equilibrium correlation Green's functions on the basis of a Boltzmann equation. // Theor. Math. Phys., 1990, vol. 82, No. 2, p. 200-212.

97. Bosse J., Götze W., Lücke M. Mode-coupling theory of simple classical liquids. // Phys. Rev. A, 1978, vol. 17, No. 1, p. 434-454.

98. Zubarev D.N., Morozov V.G. Statistical mechanics of nonlinear hydrodynamic fluc- 
tuations. // Physica A, 1983, vol. 120, No. 3-4, p. 411-467.

99. Adjemjan L.Ts., Grinin A.P., Kuni F.M. Time asymptotic behaviour of the kinetic cores of the linear hydrodynamics. // Teor. Mat. Fiz., 1975, vol. 24, No. 2, p. 813-819.

100. Zubarev D.N., Morozov V.G., Omelyan I.P., Tokarchuk M.V. Unification of the kinetic and hydrodynamic approaches in the theory of dense gases and liquids. // Teor. Mat. Fiz., 1992, vol. 96, No. 3, p. 997-1012.

\title{
Узагальнена статистична гідродинаміка флюїдів: підхід узагальнених колективних мод
}

\author{
І.М. Мриглод \\ Інститут фізики конденсованих систем НАН України, \\ 290011, Львів-11, вул. Свєнціцького, 1 \\ Отримано 29 червня 1998 р.
}

Метою цієї оглядової роботи є обговорення недавніх досягнень в узагальненій гідродинаміці густих газів та рідин. Робиться спроба представлення відомих раніше результатів у контексті перспектив нерівноважної теорії рідин, при цьому основний наголос робиться на формалізмі узагальнених колективних мод. Цей підхід дозволяє отримати узгоджений опис динаміки флюїдних систем у широкій області зміни хвильового вектора та частоти і провести розрахунки часових кореляційних функцій та узагальнених коефіцієнтів переносу в рамках єдиної схеми. Розгляд розпочинається із викладу основних рівнянь теорії. Потім обговорюються застосування методу узагальнених колективних мод до вивчення динамічних властивостей різних систем: ленард-джонсівської рідини, металічного цезію, бінарних сумішей і полярних рідин. Показано, як метод може бути поширений для дослідження магнітних рідин та багатокомпонентних сумішей. Для ілюстрації представлено результати, знайдені теоретично для спектрів узагальнених колективних мод у різних системах. Наведені експериментальні факти, що свідчать у користь спостережуваних пропагаторних кінетичних мод, знайдених у теорії. Коротко обговорюються ефекти взаємодії мод. Обговорюються також питання взаємозв'язку пропонованого підходу з іншими методами, що застосовуються в теорії рідин.

Ключові слова: узагальнена гідродинаміка, колективні збудження, флюїди, суміші, часова кореляційна функція

PACS: 05.20.-y, 05.60.+w, 05.70.Ln 